\title{
Hamilton-Jacobi Wave Theory in Manifestly-Covariant Classical and Quantum Gravity
}

\author{
Claudio Cremaschini ${ }^{1, *(D)}$ and Massimo Tessarotto ${ }^{2,3}$ (D) \\ 1 Institute of Physics and Research Center for Theoretical Physics and Astrophysics, Faculty of Philosophy \\ and Science, Silesian University in Opava, Bezručovo nám.13, CZ-74601 Opava, Czech Republic \\ 2 Department of Mathematics and Geosciences, University of Trieste, Via Valerio 12, 34127 Trieste, Italy; \\ maxtextss@gmail.com \\ 3 Institute of Physics, Faculty of Philosophy and Science, Silesian University in Opava, Bezručovo nám.13, \\ CZ-74601 Opava, Czech Republic \\ * Correspondence: claudiocremaschini@gmail.com
}

Received: 17 February 2019; Accepted: 19 April 2019; Published: 24 April 2019

check for updates

\begin{abstract}
The axiomatic geometric structure which lays at the basis of Covariant Classical and Quantum Gravity Theory is investigated. This refers specifically to fundamental aspects of the manifestly-covariant Hamiltonian representation of General Relativity which has recently been developed in the framework of a synchronous deDonder-Weyl variational formulation (2015-2019). In such a setting, the canonical variables defining the canonical state acquire different tensorial orders, with the momentum conjugate to the field variable $g_{\mu \nu}$ being realized by the third-order 4-tensor $\Pi_{\mu v}^{\alpha}$. It is shown that this generates a corresponding Hamilton-Jacobi theory in which the Hamilton principal function is a 4-tensor $S^{\alpha}$. However, in order to express the Hamilton equations as evolution equations and apply standard quantization methods, the canonical variables must have the same tensorial dimension. This can be achieved by projection of the canonical momentum field along prescribed tensorial directions associated with geodesic trajectories defined with respect to the background space-time for either classical test particles or raylights. It is proved that this permits to recover a Hamilton principal function in the appropriate form of 4-scalar type. The corresponding Hamilton-Jacobi wave theory is studied and implications for the manifestly-covariant quantum gravity theory are discussed. This concerns in particular the possibility of achieving at quantum level physical solutions describing massive or massless quanta of the gravitational field.
\end{abstract}

Keywords: covariant quantum gravity; Hamilton equations; Hamilton-Jacobi theory; wave theory; massive/massless gravitons

PACS: 03.65.Ca; 03.65.Ta

\section{Introduction}

This paper is part of a continuing long-term collaborative research effort about theoretical foundations and principles of Classical and Quantum Gravity. In particular, in this paper, the axiomatic geometric structure laying at the basis of both Covariant Classical and Quantum Gravity Theories (respectively CCG and CQG theories) is investigated. This refers specifically to a crucial aspect of the so-called standard formulation of General Relativity (SF-GR), i.e., the Einstein field equations, and which is also of key importance for establishing a corresponding self-consistent quantization theory, namely Quantum Gravity. More precisely, this concerns the Hamiltonian properties of the Einstein field equations recently achieved in the context of a reduced-dimensional variational deDonder-Weyl approach [1-9] and based on a synchronous variational formulation [10]. This refers to the discovery 
of a new classical Hamiltonian representation associated with SF-GR, which departs from the customary realizations typically considered in past literature and which ultimately date back to the non manifestly-covariant formalism introduced by Dirac [11,12]. In fact, the new Hamiltonian system is found to exhibit three key characteristic properties, namely it is:

- manifestly covariant, i.e., it can be set in 4-tensor form;

- unconstrained, i.e., the same Hamiltonian system can be expressed in terms of an independent set of canonical variables;

- variational. Indeed, as typical of classical Hamiltonian systems occurring in classical mechanics, one can show that also the new abstract Hamiltonian system can be determined via a suitable path-integral variational principle.

In contrast, if the Hamiltonian systems are not set in tensor form, so that the canonical variables and the Hamiltonian density are not 4-tensors, then the property of manifest covariance is violated. In addition, the same canonical variables are not independent, being generally subject to constraints between generalized Lagrangian coordinates and canonical momenta. Hence, while the variational property indicated above simply remains non-applicable in such a context, also a true Hamiltonian structure is effectively missing. Indeed, it is well-known that even certain classes of non-Hamiltonian systems can always be reduced to suitably-constrained Hamiltonian systems (sic). The consequences of such a type of setting are serious. "Inter alia": (1) At the classical level the correct gauge properties of SF-GR, which usually hold in classical field theory, are now prohibited (see Ref. [10]); (2) Standard canonical quantization methods become inapplicable; (3) Both at classical and quantum levels, the so-called Principle of objectivity is violated, namely the fundamental requisite of retaining the same form in arbitrary coordinate systems (GR-frames) is not fulfilled any more.

These statements, in view of their actual impact and their conceptual implications, involve an analysis of the abstract, i.e., geometrical, structure of space-time. The latter can be achieved in principle via either continuum or discrete representations. The task is not simply a tutorial to CQG- (and CCQ-) theory.

In fact, challenging mathematical questions are involved which have to be established on rigorous grounds and correspondingly physical interpretations. Of foremost importance in particular is the first property (i.e., the manifest covariance) indicated above, which realizes a symmetry property holding in the context of both Classical and Quantum Gravity. In fact, although the adoption of special coordinate systems (GR-frames) is always legitimate, the differential-manifold structure of space-time makes its structure frame-independent, i.e., symmetric with respect to the group of local point transformations

$$
r^{\mu} \rightarrow r^{\prime \mu}=r^{\prime \mu}(r)
$$

mapping between different general relativistic (GR) frames $r \equiv\left\{r^{\mu}\right\}$ and $r^{\prime} \equiv\left\{r^{\prime \mu}\right\}$.This implies, in turn, the mandatory consequence that all physical laws which are assumed to hold everywhere in the whole universe should retain their (tensor) form independent of the GR-frame and hence be intrinsically manifestly-covariant.

These premises motivate the investigation of a number of related topics which concern in particular:

- The space-time transformation properties with respect to the group of local point transformations, i.e., coordinate transformations, and the consistency of current realizations adopted for classical and quantum gravity theories with respect to the principle of manifest covariance [13-17]. The issue pertains both to the identification of the classical Hamiltonian and Hamilton-Jacobi structures of General Relativity, as well as to a corresponding prescription of the physical postulates at the basis of a quantum mechanical description of space-time and canonical quantization in terms of continuum or discrete space-time configurations [18-20]. 
- Trajectory-based dynamics of the classical and quantum gravitational field and the statistical foundations of quantum space-time dynamics, including the validity of Heisenberg inequalities, thermodynamical characterization and entropic principles [21-23].

- The symmetry properties of space-time related to the emergent gravity phenomenon, whereby certain physical observables/characteristics of classical General Relativity follow from quantum gravity theory. These concern both the prescription of the local-coordinate value of the space-time metric tensor, via a suitable quantum expectation value, as well as the establishment of the very functional form of the General Relativity field equations [22,24].

Based on these considerations, in this paper, properties of the mathematical structure of the manifestly-covariant Hamiltonian and Hamilton-Jacobi theories of classical General Relativity, both set at the foundations of CCQ- and CQG-theories, are investigated.

For this purpose, the initial setting of the theory will be based (as originally first pointed out in Ref. [10]) on a variational formulation of the Einstein field equations which belongs to the class of so-called deDonder-Weyl approaches to continuum field dynamics [1-9] (and for this reason referred to here as deDonder-Weyl synchronous-variational approach to GR). Its peculiar characteristic is, in fact, that of leaving invariant the space-time volume element in the variational functional, a feature which is achieved thanks to the adoption of a so-called synchronous variational principle. A basic property of such a formulation is that it fulfills the property of manifest covariance of the theory, i.e., the consistency with the principles of covariance and manifest covariance, while at the same time for appropriate boundary conditions the corresponding variational Euler-Lagrange equations should exactly coincide with the Einstein field equations. As such, the same approach should provide the proper framework for the identification of the canonical structure underlying classical General Relativity, a feature that differs from alternative non-manifestly covariant approaches to the problem built upon preliminary space-time foliations [25-28].

The synchronous variational principle is characterized by the adoption of superabundant variables, leading to the distinction between a continuum background metric tensor $\widehat{g}_{\mu v}$ and a variational field $g_{\mu v}$. In particular, while performing the variations in the variational functional, the components of $g_{\mu v}$ (and the corresponding conjugate momenta) are considered independent while their extremal values (appearing in the Euler-Lagrange equations) are then required to coincide with $\widehat{g}_{\mu v}$. Concerning the notation, in the paper, all hatted quantities refer to functions of the background tensor $\widehat{g}_{\mu v}$. According to this picture, the background metric tensor $\widehat{g}_{\mu v}$ raises/lowers tensor indices, has vanishing covariant derivatives and yields the geometric properties of the space-time, e.g., the Ricci curvature tensor and the Christoffel symbols. Instead, the variational tensor $g_{\mu \nu}$ is associated with the physical properties of gravitational field expressed through kinetic and potential contributions in the corresponding Lagrangian function. In this sense, $g_{\mu \nu}$ has no geometrical interpretation, and therefore it does not raise or lower indices, and its dynamical equations are determined by the Euler-Lagrange equations following from the synchronous variational principle. It is then found that, when passing from classical to quantum descriptions, the variational field $g_{\mu \nu}$ identifies the quantum gravitational field (i.e., the quantum observable) which dynamically evolves over $\widehat{g}_{\mu v}$ according to a defined quantum wave equation (CQG-wave equation) [20].

A characteristic feature which is expected to occur in such a setting (i.e., in the context of the deDonder-Weyl synchronous-variational approach) is that the 4-tensor canonical variables which define the canonical state acquire different tensorial orders. Thus, once the generalized coordinates are identified with the second-order symmetric 4-tensor $g_{\mu v}$, its conjugate momentum is found to be realized by a third-order 4 -tensor $\Pi_{\mu v}^{\alpha}$. As a consequence, the resulting canonical state turns out to be represented by the mixed-order set of extended variables $x \equiv\left\{g_{\mu v}, \Pi_{\mu v}^{\alpha}\right\}$, with the corresponding classical Hamiltonian structure being given by $\{x, H\}$, with $H$ a suitable Hamiltonian density function. Because of the difference of tensorial orders between canonical variables, this is referred to here as extended Hamiltonian formulation of GR [18]. Despite this feature, this suggests that thanks to the 
formal analogy of the canonical theory with the Hamiltonian formulation of classical mechanics, the development of a theory of canonical transformation for the continuum fields $\{x, H\}$ should still be permitted. These include, as a relevant realization, a so-called canonical transformation of the second type (according to the name usually given in classical mechanics), which leads to the realization of a Hamilton-Jacobi theory corresponding to the Hamiltonian formulation [19]. Basic characteristic, however, is that of exhibiting a crucial non-uniqueness feature: i.e., the canonical equations fulfilled by the extended canonical state $x$ generally do not uniquely determine the canonical momentum $\Pi_{\mu v}^{\alpha}$.

It must be stressed that, a priori, the existence of such a theory as well as the equivalence between Hamilton and Hamilton-Jacobi theories are not properties that necessarily should be expected to hold. Indeed, the actual proof of validity of such a relationship remains to be ascertained. The task is accomplished in the present paper. This provides a coherent theoretical setting for the classical continuum gravitational field in which Lagrangian, Hamiltonian and Hamilton-Jacobi theories are obtained and mutually implied from each other. In particular, stemming from the extended Hamiltonian theory, it is shown that canonical transformations generate a corresponding extended Hamilton-Jacobi theory, namely in which the Hamilton principal function is represented by a 4-tensor $S^{\alpha}$ (rather than a scalar as is customary in this type of problem).

Beyond these theoretical outcomes, there arises the conceptual problem of having Hamiltonian and Hamilton-Jacobi theories for the gravitational field which are cast in a form suitable for attempting their quantum representation in a form appropriate for the development of corresponding manifestly-covariant quantum gravity theories [20,21]. It turns out, however, that extended formulations (of the type indicated above) are not suitable for the task because of the non-uniqueness of the third-order tensor canonical momentum. In addition, a further issue concerns the implementations, in the framework of manifestly-covariant relativistic theories, of standard quantization methods which are already well-established in quantum mechanics [29-31]. To reach this goal and to afford an analogous physical interpretation of the corresponding quantum theory, in fact, the requirement is that the canonical variables should have the same tensorial dimension, with the Hamilton principal function being represented by a single 4 -scalar real function. The mathematical procedure that is adopted here in order to achieve this kind of representation is based on the introduction of a suitable projection operator $\Sigma$. More precisely, $\Sigma$ is prescribed in such a way to act on the extended canonical momentum field and the Hamilton principal function in such a way to lower their tensorial order, while leaving unchanged the underlying canonical structure of the theory. As a result, the action of $\Sigma$ is found to produce corresponding reduced Hamiltonian and reduced Hamilton-Jacobi theories which are proved to be equivalent to the extended ones (and at the same time to be mutually implied by each other).

After listing a number of physical and mathematical pre-requisites that such an operator must satisfy, the latter is finally identified with a 4 -vector $\Sigma \equiv \Sigma^{\alpha}$, so that the projection is interpreted as occurring along a prescribed tensorial direction given by the same $\Sigma^{\alpha}$. Only two physically-admissible choices of $\Sigma^{\alpha}$ are identified, whereby respectively $\Sigma^{\alpha}=t^{\alpha}$ or $\Sigma^{\alpha}=k^{\alpha}$. More precisely, in the first case, the 4 -vector $t^{\alpha}$ is the tangent 4 -vector to sub-luminal geodesic trajectories of the background space-time $\widehat{g}_{\mu \nu}$ associated with massive test particles and characterized by proper-time arc-length $d s^{2}=\widehat{g}_{\mu \nu} d r^{\mu} d r^{v}$, where by definition $t^{\alpha} \equiv \frac{d r^{\alpha}}{d s}$. Instead, in the second case, the 4-vector $k^{\alpha}$ corresponds to the wave 4-vector associated with light-ray geodetics and having by definition null magnitude (i.e., such that $k^{\alpha} k_{\alpha}=0$ ) [14]. Only the choice $\Sigma^{\alpha}=t^{\alpha}$ is shown to be acceptable, which allows one to express the Hamilton equations as evolution equations in terms of proper-time evolution parameter $s$, and to recover as well a Hamilton principal function in the appropriate form of 4-scalar type.

The reduced Hamilton-Jacobi wave theory for the two choices of projection operator identified above is studied and conceptual implications for the manifestly-covariant quantum gravity theory are discussed. This concerns in particular the possibility of achieving at quantum level physical solutions describing either massive or massless quanta of the gravitational field. As a notable outcome, it is proved that general representations for the Hamilton principal function which combine both $t^{\alpha}-$ 
and $k^{\alpha}$-projections can in principle coexist, with the latter describing luminal waves propagating at the speed of light $c$. However, this remains a sort of gauge-type contribution, in the sense that the combination of massive and massless wave terms in the Hamilton principal function have a meaning only for the classical Hamilton-Jacobi theory. Indeed, by themselves the massless terms cancel out identically from the classical theory and only the massive term survives, which can be finally accounted for by the corresponding quantum gravity theory. The result is that a quantum dynamics built on the manifestly-covariant continuum canonical theory can only treat wave-like solutions corresponding to massive gravitons. Therefore, according to this formalism, although the reduced representation can include in principle both massive and massless classical wave contributions, the massless waves have only a classical connotation and they cannot pertain the quantum gravity theory stemming from the GR canonical formalism. This conclusion provides a theoretical support to the quantum gravity theory early developed in Refs. [19-22,24], proving that the description of massive quantum gravitons is indeed a characteristic feature of the manifestly-covariant theory and a strict consequence of its formalism. The present outcome also gives completeness to the canonical formalism of the theory and reinforces the strong symmetry link existing between the continuum classical canonical framework and the corresponding discrete quantum representation of the theory of gravitational field.

The scheme of the paper is as follows. In Section 2, the extended Hamiltonian formulation is introduced, while, in Section 3, the theory of canonical transformations and related extended Hamilton-Jacobi theory are developed, proving the equivalence between the Hamiltonian and Hamilton-Jacobi problems for the continuum gravitational field. In Sections 4 and 5, respectively, the issues about the search of reduced Hamiltonian and Hamilton-Jacobi theories are discussed. In Section 6, the concept of projection operator is introduced, which relates extended and reduced canonical formulations, and the physical and mathematical requisites that it must satisfy are given. Section 7 deals with the possibility of describing wave-like solutions in the framework of the Hamilton-Jacobi theory, which can correspond to either massless or massive graviton particles after quantization. Main conclusions of the work are reported in Section 8. Finally, in Appendices A and B, mathematical details of calculations are given for completeness.

\section{Extended Hamiltonian Formulation}

In this section, the fundamental content of the manifestly-covariant Hamiltonian theory of classical GR is formulated in the framework of a deDonder-Weyl synchronous-variational approach to GR, inspired by Refs. [1,2] and originally first developed in Ref. [10] for the case of a stationary background metric tensor of the type $\widehat{g}_{\mu \nu}=\widehat{g}_{\mu \nu}(r)$. The approach is extended here to the more general and physically-significant case of a non-stationary setting of the form $\widehat{g}_{\mu \nu}=\widehat{g}_{\mu \nu}(r, s) \equiv \widehat{g}_{\mu \nu}(s)$ (see Refs. [22,24]). The Hamiltonian theory is found to be represented by the classical Hamiltonian structure $\{x, H\}$, which is formed by an appropriate extended 4-tensor canonical state $x \equiv\left\{g_{\mu v}, \Pi_{\mu \nu}^{\alpha}\right\}$ and a suitable 4-scalar Hamiltonian density $H(x, \widehat{x}(s), r, s)$, where $g_{\mu v}$ represents the Lagrangian coordinate expressed by the second-order variational field 4 -tensor of the gravitational field and $\Pi_{\mu \nu}^{\alpha}$ is its conjugate third-order momentum 4-tensor. Because the coordinate field and its conjugate momentum have different tensorial orders, this Hamiltonian formulation is referred to in the following as extended Hamiltonian theory. It provides the reference background for the subsequent development of the extended Hamilton-Jacobi theory and also of corresponding reduced Hamiltonian and H-J theories characterized by a canonical state in which field variables and conjugate momenta have the same tensorial orders.

Let us first introduce the variational Lagrangian density for the non-vacuum Einstein field equations in the presence of cosmological constant (see also Ref. [10]), which is given by the 4 -scalar function

$$
L\left(Z, \widehat{\nabla}_{\mu} Z, \widehat{Z}\right)=L_{G}\left(Z, \widehat{\nabla}_{\mu} Z, \widehat{Z}\right)+L_{\Lambda}(Z, \widehat{Z})+L_{F}(Z, \widehat{Z})
$$


where for shortness of notation here $Z=g_{\mu \nu}$ is the variational field coordinate, $\widehat{Z}=\widehat{g}_{\mu \nu}$ denotes the background metric tensor and $\hat{\nabla}_{\mu} Z$ is the covariant derivative of $g_{\mu v}$ written with respect to $\widehat{g}_{\mu \nu}$. The contributions $L_{G}$ and $L_{\Lambda}$ on the rhs of Equation (2) refer respectively to the gravitational and cosmological-constant Lagrangian terms, which are defined as follows (see also Ref. [20])

$$
\begin{gathered}
L_{G}\left(Z, \widehat{\nabla}_{\mu} Z, \widehat{Z}\right)=-\kappa g^{\mu v} \widehat{R}_{\mu v} h(Z, \widehat{Z})+\kappa \frac{1}{2} \widehat{\nabla}^{\alpha} g_{\mu \nu} \widehat{\nabla}_{\alpha} g^{\mu v}, \\
L_{\Lambda}(Z, \widehat{Z})=-2 \kappa \Lambda h(Z, \widehat{Z}) .
\end{gathered}
$$

Instead, the term $L_{F}$ in Equation (2) denotes the Lagrangian contribution carried by external fields different from the gravitational one, which can be prescribed in non-vacuum configurations and generates the stress-energy tensor $T_{\mu v}$ according to the customary variational derivation [14]. The notations in the previous equations are standard ones, namely $\widehat{R}_{\mu v}$ is the Ricci tensor defined with respect to the background metric tensor $\widehat{g}_{\mu \nu}, \kappa$ identifies the constant $\kappa=\frac{c^{3}}{16 \pi G}, \Lambda$ is the cosmological constant and $h(Z, \widehat{Z})$ is the 4 -scalar multiplicative factor

$$
h(Z, \widehat{Z}) \equiv\left(2-\frac{1}{4} g^{\alpha \beta} g_{\alpha \beta}\right)
$$

where by definition $g^{\alpha \beta} g_{\alpha \beta} \neq \delta_{\alpha}^{\alpha}$ for variational curves.

The 4-scalar (2) is the Lagrangian function of the synchronous variational principle. It means that the quantity

$$
\left.\frac{\delta L\left(Z, \widehat{\nabla}_{\alpha} Z, \widehat{Z}\right)}{\delta g^{\mu \nu}}\right|_{g_{\mu v}=\widehat{g}_{\mu v}}=0
$$

yields the classical Einstein field equations as extremal equations evaluated for $g_{\mu \nu}=\widehat{g}_{\mu v}$, where here $\delta$ denotes the synchronous variational operator which leaves invariant the background metric tensor $\widehat{g}_{\mu \nu}$ and the 4 -volume element $d \Omega \equiv d^{4} x \sqrt{-|\widehat{g}|}$, namely such that identically $\delta d \Omega=0$. It must be stressed that the synchronous variational principle is distinguished from customary asynchronous variational principles of GR available in the literature for which instead $\delta_{\text {async }} d \Omega \neq 0$. The synchronous condition $\delta d \Omega=0$ is the basis of the manifestly-covariance property of $L\left(Z, \widehat{\nabla}_{\alpha} Z, \widehat{Z}\right)$, a feature which in turn is gained thanks to the introduction of the variational function $h(Z, \widehat{Z})$ and the adoption of superabundant variables, distinguishing between the background metric tensor $\widehat{g}_{\mu v}$ and the variational field $g_{\mu v}$, the two being required to coincide only for extremal field equations.

Given the Lagrangian formulation, it is then possible to define the canonical momentum conjugate to the Lagrangian coordinate $g_{\mu v}$, which in the present notation is given by the third-order 4-tensor

$$
\Pi_{\mu v}^{\alpha}=\frac{\partial L\left(Z, \hat{\nabla}_{\mu} Z, \widehat{Z}\right)}{\partial\left(\widehat{\nabla}_{\alpha} g^{\mu v}\right)}=\kappa \widehat{\nabla}^{\alpha} g_{\mu \nu} .
$$

The momentum $\Pi_{\mu v}^{\alpha}$ is symmetric in the lower indices, in the sense that $\Pi_{\mu v}^{\alpha}=\Pi_{v \mu}^{\alpha}$, it is non-vanishing since $g_{\mu v} \neq \widehat{g}_{\mu v}$ and must be considered as non-extremal. On the other hand, the extremal value of $\Pi_{\mu v}^{\alpha}$, namely $\widehat{\Pi}_{\mu v}^{\alpha}=\kappa \widehat{\nabla}^{\alpha} \bar{g}_{\mu v}$, vanishes identically. We further notice that, thanks to the quadratic dependence of the Lagrangian with respect to the generalized velocity, Equation (7) is invertible, yielding a relationship between momenta and generalized velocities analogous to the one occurring in relativistic particle dynamics. 
Given these premises, one can proceed formulating the manifestly-covariant Hamiltonian theory of GR. More precisely, the Hamiltonian density $H=H(x, \widehat{x})$ associated with the Lagrangian $L\left(Z, \widehat{\nabla}_{\mu} Z, \widehat{Z}\right)$ is provided, as in classical mechanics, by the Legendre transform

$$
L\left(Z, \widehat{\nabla}_{\mu} Z, \widehat{Z}\right) \equiv \Pi_{\mu v}^{\alpha} \widehat{\nabla}_{\alpha} g^{\mu \nu}-H(x, \widehat{x}) .
$$

In the Lagrangian function (2), the contribution due to the momentum $\Pi_{\mu v}^{\alpha}$ is only present in the term $L_{G}\left(Z, \widehat{\nabla}_{\mu} Z, \widehat{Z}\right)$. The latter one, when written in canonical variables, is such that $L_{G}\left(Z, \widehat{\nabla}_{\mu} Z, \widehat{Z}\right)=$ $L_{G}(x, \widehat{x})$, where

$$
L_{G}(x, \widehat{x})=-\kappa g^{\mu \nu} \widehat{R}_{\mu v} h+\frac{1}{2 \kappa} \Pi_{\mu v}^{\alpha} \Pi_{\alpha}^{\mu \nu} .
$$

The remaining contributions $L_{\Lambda}(Z, \widehat{Z})$ and $L_{F}(Z, \widehat{Z})$ are only field-coordinate dependent, and therefore their expression is not affected by the Legendre transform. The Lagrangian function expressed in the canonical variables then is written

$$
L(x, \widehat{x})=L_{G}(x, \widehat{x})+L_{\Lambda}(Z, \widehat{Z})+L_{F}(Z, \widehat{Z}) .
$$

As a result, the Hamiltonian density $H(x, \widehat{x})$ is given by

$$
H(x, \widehat{x})=H_{G}(x, \widehat{x})-L_{\Lambda}(Z, \widehat{Z})-L_{F}(Z, \widehat{Z}),
$$

where in particular

$$
H_{G}(x, \widehat{x}) \equiv \frac{1}{2 \kappa} \Pi_{\mu \nu}^{\alpha} \Pi_{\alpha}^{\mu v}+\kappa g^{\mu v} \widehat{R}_{\mu v} h .
$$

Introducing then the Hamiltonian action functional

$$
S_{H}(x, \widehat{x})=\int d \Omega L(x, \widehat{x}),
$$

where $L(x, \widehat{x})$ is given by Equation (10), the synchronous Hamiltonian variational principle $\delta S_{H}(x, \widehat{x})=0$ is required to hold for arbitrary independent synchronous variations $\delta g^{\mu v}$ and $\delta \Pi_{\mu \nu}^{\alpha}$ in the respective functional classes. We omit here the mathematical details of the proof of this statement, as they can be found in Ref. [18]. The corresponding variational derivatives yield the so-called extended continuum Hamilton equations

$$
\begin{aligned}
\frac{\delta S_{H}(x, \widehat{x})}{\delta g^{\mu \nu}} & \equiv-\frac{\partial H(x, \widehat{x})}{\partial g^{\mu \nu}}-\hat{\nabla}_{\alpha} \Pi_{\mu \nu}^{\alpha}=0, \\
\frac{\delta S_{H}(x, \widehat{x})}{\delta \Pi_{\mu \nu}^{\alpha}} & \equiv \hat{\nabla}_{\alpha} g^{\mu v}-\frac{\partial H(x, \widehat{x})}{\partial \Pi_{\mu \nu}^{\alpha}}=0
\end{aligned}
$$

to be supplemented by suitable boundary conditions for the extended canonical state $x \equiv\left\{g_{\mu v}, \Pi_{\mu \nu}^{\alpha}\right\}$. These equations can be equivalently cast in terms of local 4-vector Poisson brackets (see Appendix A, Equations (A8) and (A9)). Written explicitly, the same equations are realized by the PDEs

$$
\begin{aligned}
\hat{\nabla}_{\alpha} \Pi_{\mu v}^{\alpha} & =-\frac{\partial H(x, \widehat{x})}{\partial g^{\mu v}}, \\
\hat{\nabla}_{\alpha} g^{\mu v} & =\frac{1}{\kappa} \Pi_{\alpha}^{\mu v},
\end{aligned}
$$


so that the second one recovers as usual the definition of the canonical momentum. This set of equations is equivalent to the Euler-Lagrange equation identified with the Einstein field equation. In fact, the following property holds.

Property 1. (Extremal property)—Provided $g_{\mu v}$ is identified with the extremal solution $\widehat{g}_{\mu v}$, then respectively $\widehat{\Pi}_{\mu v}^{\alpha}$ and $\widehat{g}_{\alpha \beta}$ satisfy identically the equation $\widehat{\Pi}_{\mu v}^{\alpha}=0$ and the Einstein equations.

Proof. In fact, if one requires $g_{\mu v} \equiv \widehat{g}_{\mu v}$, it follows that $\widehat{\nabla}_{\alpha} g^{\mu v} \equiv 0$. This warrants that identically $\widehat{\Pi}_{\mu v}^{\alpha}=0$ while elementary algebra shows that the first tensor Equation (16) recovers the Einstein equations.

This completes the derivation of the extended Hamiltonian theory of GR and, thanks to Property 1 , the proof of its formal connection with the SF-GR.

However, the additional notable feature of the same equations should be pointed out.

Property 2. (Non-uniqueness property)—Provided $g_{\mu \nu}$ differs from the extremal solution $\widehat{g}_{\mu v}$, it follows that the solution of Equations (16) and (17) is generally non-unique.

Proof. Let us show, in fact, that Equations (16) and (17) generally do not uniquely determine the extended canonical momentum $\Pi_{\mu \nu}^{\alpha}$. This conclusion follows from the first PDE indicated above (i.e., Equation (16)) by noting that the same equation does not prescribe uniquely the 4th-order tensor covariant derivatives $\widehat{\nabla}_{\alpha} \Pi_{\mu \nu}^{\beta}$ but only their saturation $\hat{\nabla}_{\alpha} \Pi_{\mu \nu}^{\alpha}$.

Notice that this conclusion is not at variance with Property 1 . Nevertheless, the issue of non-uniqueness for Equations (16) and (17) remains virtually still open for more general (i.e., non-vanishing) boundary conditions for $\widehat{\Pi}_{\mu v}^{\alpha}$.

\section{Canonical Transformations and Extended Hamilton-Jacobi Theory}

In this section, we pose the problem of the construction of canonical transformations which preserve the Hamiltonian structure of the continuum Equations (16) and (17) (see also Equations (A8) and (A9) reported for convenience in Appendix A), together with the related derivation of a manifestly-covariant extended Hamilton-Jacobi theory for the classical gravitational field corresponding to the extended Hamiltonian formulation. In doing this, we shall regard the tensor fields $\widehat{g}_{\alpha \beta}$ and $g_{\alpha \beta}$ as independent. In such a setting, the canonical transformations are defined in terms of a local diffeomorphism of the form

$$
\begin{aligned}
& x \rightarrow X=X(x, r), \\
& X \rightarrow x=x(X, r),
\end{aligned}
$$

where $x \equiv\left\{g^{\beta \gamma}, \Pi_{\beta \gamma}^{\alpha}\right\}$ and $X \equiv\left\{Q^{\beta \gamma}, P_{\beta \gamma}^{\alpha}\right\}$. More generally, however, one can always allow $X$ to depend also on $\widehat{x} \equiv\left\{\widehat{g}_{\alpha \beta}, \widehat{\Pi}_{\mu \nu}^{\alpha}=0\right\}$, namely to be of the form $X=X(x, \widehat{x}, r)$. Furthermore, we denote by $\widehat{X}=X(\widehat{x}, \widehat{x}, r)$. Notice that the transformation (18) may not be defined globally on the open set $D^{4}$ and in particular may depend also on how the boundary set $\partial D^{4}$ is prescribed. The reason is that the continuum Hamilton equations themselves may not be defined on $\partial D^{4}$, due to possible discontinuities of the canonical fields arising on such a set. By definition, the map transforms the Lagrangian density $L(x, \widehat{x}) \equiv L\left(Z, \widehat{\nabla}_{\mu} Z, \widehat{Z}\right)$ given by Equation (8) in terms of the equation

$$
L(x, \widehat{x})=L_{T}(X, \widehat{X}, r)+\widehat{\nabla}_{\alpha} S^{\alpha}
$$


where $L_{T}(X, \widehat{X}, r)$ is the transformed Lagrangian which is taken of the form

$$
L_{T}(X, \widehat{X}, r)=P_{\mu v}^{\alpha} \widehat{\nabla}_{\alpha} Q^{\mu v}-K(X, \widehat{X}, r),
$$

while $S^{\alpha}$ can be interpreted as an arbitrary 4-vector mixed-variable generating function (gauge function) for the transformation (18), which can for example be assumed to be a smooth function of the variables $(x, X, r)$. Moreover, notice that, in principle, $S^{\alpha}$ can contain arbitrary dependences in terms of both $\widehat{x}$ and $\hat{X}$. In fact, due to the gauge property of the Lagrangian density, the gradient operator $\hat{\nabla}_{\alpha}$ in Equation (20) acts only on the variables $(x, X, r)$ contained in $S^{\alpha}$. In particular, omitting for brevity the latter dependences, we can always prescribe $S^{\alpha}$ in terms of the Legendre transformation

$$
S^{\alpha}=-Q^{\mu v} P_{\mu v}^{\alpha}+S_{2}^{\alpha}\left(g^{\beta \gamma}, P_{\beta \gamma}^{(\alpha)}, r\right)
$$

where we notice that in the second term on the rhs the internal repeated index $\alpha$ is dummy. As a consequence of the previous equation, Equation (20) requires that the transformed Hamiltonian density $K$ must be allowed to be also explicitly dependent on the position 4-vector $r$. From Equations (20) and (22) it also follows that

$$
\Pi_{\mu \nu}^{\alpha} \widehat{\nabla}_{\alpha} g^{\mu v}-H(x, \widehat{x})=P_{\mu \nu}^{\alpha} \widehat{\nabla}_{\alpha} Q^{\mu v}-K(X, \widehat{X}, r)+\widehat{\nabla}_{\alpha} S^{\alpha} .
$$

Invoking Equation (22), one obtains:

$$
\begin{aligned}
\hat{\nabla}_{\alpha} S^{\alpha}= & -Q^{\mu \nu} \widehat{\nabla}_{\alpha} P_{\mu \nu}^{\alpha}-P_{\mu \nu}^{\alpha} \hat{\nabla}_{\alpha} Q^{\mu \nu}+\hat{\nabla}_{\alpha} g^{\mu \nu} \frac{\partial S_{2}^{\alpha}\left(g^{\beta \gamma}, P_{\beta \gamma^{\prime}}^{\alpha} r\right)}{\partial g^{\mu \nu}} \\
& +\hat{\nabla}_{\alpha} P_{\beta \gamma}^{\alpha} \frac{\partial S_{2}^{\lambda}\left(g^{\beta \gamma}, P_{\beta \gamma^{\prime}}^{\lambda}, r\right)}{\partial P_{\beta \gamma}^{\lambda}}+\left.\hat{\nabla}_{\alpha} S_{2}^{\alpha}\left(g^{\beta \gamma}, P_{\beta \gamma^{\prime}}^{\alpha} r\right)\right|_{\left(g^{\beta \gamma}, P_{\beta \gamma}^{\alpha}\right)} .
\end{aligned}
$$

Due to the assumption of a dummy internal index $(\alpha)$, in the previous terms, the summation must be intended on all the repeated indices (which include now also $\alpha$ ). The arbitrariness of $\widehat{\nabla}_{\alpha} g^{\mu v}$ and $\widehat{\nabla}_{\alpha} P_{\beta \gamma}^{\alpha}$ then implies the following transformation equations:

$$
\begin{aligned}
& \Pi_{\mu \nu}^{\alpha}=\frac{\partial S_{2}^{\alpha}\left(g^{\beta \gamma}, P_{\beta \gamma}^{(\alpha)}, r\right)}{\partial g^{\mu \nu}}, \\
& Q^{\mu v}=\frac{\partial S_{2}^{\alpha}\left(g^{\beta \gamma}, P_{\beta \gamma^{\prime}}^{\alpha} r\right)}{\partial P_{\mu \nu}^{\alpha}}, \\
& K(X, \widehat{X}, r)=H(x, \widehat{x})+\left.\widehat{\nabla}_{\alpha} S_{2}^{\alpha}\left(g^{\beta \gamma}, P_{\beta \gamma}^{\alpha}, r\right)\right|_{\left(g^{\beta \gamma}, P_{\beta \gamma}^{\alpha}\right)},
\end{aligned}
$$

where in the second equation the summation on the index $\alpha$ is understood, while in the last term of the third equation the condition on the covariant derivative must hold for each index $\alpha$. Equations (25) and (26) identify respectively half of the inverse and direct canonical transformations given respectively by Equations (19) and (18).

A fundamental feature of the continuum canonical transformations is realized by the invariance property which characterizes the 4-vector canonical Poisson brackets defined above (see Equations (A1) and (A2)). In fact, one can prove that the conditions of invariance

$$
\begin{aligned}
{[A, B]_{x, j} } & \equiv[A, B]_{X, j}, \\
{[\mathbf{A}, \mathbf{B}]_{x, j} } & \equiv[\mathbf{A}, \mathbf{B}]_{X, j}
\end{aligned}
$$


hold identically for arbitrary canonical transformations. Therefore, in the following, the labels identifying the choice of the canonical fields in the Poisson brackets can be safely omitted. We notice that canonical transformations map in one another different solutions of the continuum canonical equations. These transformations require a classification of $D^{4}$ as follows. In fact, the space-time $D^{4}$ in which the canonical fields are defined can be either bounded or unbounded (namely, coinciding with $\mathbb{R}^{4}$ ). Moreover, in both cases, the domain $D^{4}$ can be: (A) either connected or non-connected; (B) either simply connected or non simply connected. A canonical transformation of the type (18) can only be defined for connected subsets of $D^{4}$ which are either simply connected or not, while $D^{4}$ itself can be either bounded or unbounded.

Among the possible canonical transformations (produced by a mixed-variable generating function $S^{\alpha}$ which explicitly depends on the 4-position) that can be introduced and under such conditions hold in the whole set $D^{4}$, we now seek a particular one which brings to transformed fields $X$ which are constant, in the sense that

$$
\begin{aligned}
\widehat{\nabla}_{\alpha} P_{\mu v}^{\alpha} & =0, \\
\widehat{\nabla}_{\alpha} Q^{\mu v} & =0 .
\end{aligned}
$$

This requirement extends to the continuum field theory the problem originally posed in the Hamilton-Jacobi theory of classical mechanics, in which the transformed canonical variables are assumed to be constant (i.e., in the non-relativistic treatment independent of the coordinate time $t$ ). The previous constraint equations require that the corresponding Hamilton equations for $X$ are necessarily

$$
\begin{aligned}
\hat{\nabla}_{\alpha} P_{\mu v}^{\alpha} & =\left[P_{\mu v}^{\alpha}, K\right]_{\alpha} \equiv 0, \\
\widehat{\nabla}_{\alpha} Q^{\mu v} & =\left[Q^{\mu v}, K\right]_{\alpha} \equiv 0 .
\end{aligned}
$$

Since these equations must hold in the whole open set $D^{4}$, it follows that the transformed Hamiltonian $K$ cannot depend on the transformed state $X$. Hence, it can only be a function of the type $K=K(\widehat{x}, r)$, and in particular it can be proved that it $K$ can always be defined in such a way that $K(\widehat{x}, r)=0$, namely it vanishes identically.

A particular realization of Equations (32) and (33) is provided by the identification

$$
X=\widehat{x}
$$

As a consequence, both $Q^{\mu v}$ and $P_{\mu \nu}^{\alpha}$ are necessarily functions of the background metric tensor $\widehat{g}^{\alpha \beta}$ only. Therefore, from Equation (27), invoking also Equation (25), it follows

$$
H\left(g^{\mu \nu}, \frac{\partial S_{2}^{\alpha}\left(g^{\beta \gamma}, P_{\beta \gamma}^{(\alpha)}, r\right)}{\partial g^{\mu \nu}}\right)+\left.\widehat{\nabla}_{\alpha} S_{2}^{\alpha}\left(g^{\beta \gamma}, P_{\beta \gamma}^{\alpha}, r\right)\right|_{\left(g^{\beta \gamma}, P_{\beta \gamma}^{\alpha}\right)}=0,
$$

to be denoted as continuum extended Hamilton-Jacobi equation for the Hamilton 4-vector generating function $S_{2}^{\alpha}\left(g^{\beta \gamma}, P_{\beta \gamma^{\prime}}^{\alpha}, r\right)$. Notice that the solution of Equation (35) must be determined letting initially $P_{\beta \gamma}^{\alpha} \neq 0$. In the previous equation, the 4 -vector field $S_{2}^{\alpha}$ can then be prescribed by imposing also the constraint equation:

$$
Q^{\mu \nu}=\left.\frac{\partial S_{2}^{\alpha}\left(g^{\beta \gamma}, P_{\beta \gamma}^{(\alpha)}, r\right)}{\partial P_{\mu \nu}^{\alpha}}\right|_{P_{\mu \nu}^{\alpha}=0}
$$

Provided the condition on the Hessian determinant $\operatorname{det}\left|\frac{\partial^{2} S_{2}^{\alpha}\left(g^{\beta \gamma}, P_{\beta \gamma}^{(\alpha)}, r\right)}{\partial g^{\mu v} \partial P_{\mu v}^{\alpha}}\right| \neq 0$ is satisfied, Equation (36) realizes an implicit equation for $g^{\beta \gamma}$. This condition warrants the existence of the inverse 
transformation (19). In fact, once the generating function $S_{2}^{\alpha}$ is determined by solving the Hamilton-Jacobi equation, the vector Equation (36) together with Equation (25) yields the implicit function

$$
g^{\beta \gamma}=g^{\beta \gamma}\left(\widehat{g}^{\mu v}, r\right),
$$

whereby the canonical momenta are provided by Equation (25).

Let us now prove that Equation (35) is equivalent to the set of extended Hamilton equations, namely that the fields $g^{\beta \gamma}$ and $\Pi_{\alpha}^{\beta \gamma}$ satisfy the continuum canonical Equations (A8) and (A9). In fact, let us evaluate first the partial derivative of Equation (35) with respect to $g^{\beta \gamma}$, keeping both $\frac{\partial S_{2}^{\alpha}\left(g^{\beta \gamma}, P_{\beta \gamma}^{(\alpha)}, r\right)}{\partial g^{\mu \nu}}$ and $r^{\mu}$ constant. This gives

$$
\frac{\partial}{\partial g^{\beta \gamma}} H\left(g^{\mu \nu}, \frac{\partial S_{2}^{\alpha}\left(g^{\beta \gamma}, P_{\beta \gamma}^{(\alpha)}, r\right)}{\partial g^{\mu \nu}}\right)+\left.\hat{\nabla}_{\alpha}\left[\frac{\partial}{\partial g^{\beta \gamma}} S_{2}^{\alpha}\left(g^{\beta \gamma}, P_{\beta \gamma}^{\alpha}, r\right)\right]\right|_{g^{\beta \gamma}}=0 .
$$

Inspection of Equation (38) shows that this is equivalent in the whole open set $D^{4}$ to the continuum canonical Equation (A8). In fact, in Equation (38), the first term recovers the contribution given by the Poisson bracket on the rhs of Equation (A8). The second term $\frac{\partial}{\partial g^{\beta \gamma}} S_{2}^{\alpha}\left(g^{\beta \gamma}, P_{\beta \gamma^{\prime}}^{\alpha} r\right)$ instead can be identified with the initial canonical momentum $\Pi_{\beta \gamma}^{\alpha}$ which by definition must be considered independent of $g^{\beta \gamma}$ and $r^{\mu}$ as explicitly indicated above in the same equation.

Then, let us evaluate in a similar manner the partial derivative of the H-J Equation (35) with respect to $\frac{\partial S_{2}^{\alpha}\left(g^{\beta \gamma}, P_{\beta \gamma}^{(\alpha)}, r\right)}{\partial g^{\mu \nu}}$, keeping $g^{\mu v}$ and $r^{\mu}$ constant. This gives

$$
\frac{\partial}{\partial \frac{\partial S_{2}^{\lambda}\left(g^{\beta \gamma}, P_{\beta \gamma}^{(\alpha)}, r\right)}{\partial g^{\mu \nu}}} H\left(g^{\mu v}, \frac{\partial S_{2}^{\alpha}\left(g^{\beta \gamma}, P_{\beta \gamma}^{(\alpha)}, r\right)}{\partial g^{\mu \nu}}\right)+\left.\frac{\partial}{\partial \frac{\partial S_{2}^{\lambda}\left(g^{\beta \gamma}, P_{\beta \gamma}^{(\alpha)}, r\right)}{\partial g^{\mu \nu}}} \hat{\nabla}_{\alpha} S_{2}^{\alpha}\left(g^{\beta \gamma}, P_{\beta \gamma^{\prime}}^{\alpha}, r\right)\right|_{g^{\beta \gamma}}=0
$$

Invoking the identity (24), which in the present case gives

$$
\widehat{\nabla}_{\alpha} S_{2}^{\alpha}\left(g^{\beta \gamma}, P_{\beta \gamma}^{\alpha}, r\right)=\hat{\nabla}_{\alpha} g^{\beta \gamma} \frac{\partial S_{2}^{\alpha}\left(g^{\beta \gamma}, P_{\beta \gamma^{\prime}}^{\alpha} r\right)}{\partial g \beta \gamma}+\left.\widehat{\nabla}_{\alpha} S_{2}^{\alpha}\left(g^{\beta \gamma}, P_{\beta \gamma}^{\alpha}, r\right)\right|_{g \beta \gamma},
$$

it follows that the only term which depends on the partial derivative $\frac{\partial S_{2}^{\lambda}\left(g^{\beta \gamma}, P_{\beta \gamma}^{(\alpha)}, r\right)}{\partial g^{\mu \nu}}$ is the first one on the rhs of the previous equation, so that one obtains

$$
\left.\frac{\partial}{\partial \frac{\partial S_{2}^{\lambda}\left(g^{\beta \gamma}, P_{\beta \gamma}^{(\alpha)}, r\right)}{\partial g^{\mu \nu}}} \hat{\nabla}_{\alpha} S_{2}^{\alpha}\left(g^{\beta \gamma}, P_{\beta \gamma}^{\alpha}, r\right)\right|_{g^{\beta \gamma}}=-\frac{\partial}{\partial \frac{\partial S_{2}^{\lambda}\left(g^{\beta \gamma}, P_{\beta \gamma}^{(\alpha)}, r\right)}{\partial g^{\mu \nu}}} \hat{\nabla}_{\alpha} g^{\beta \gamma} \frac{\partial S_{2}^{\alpha}\left(g^{\beta \gamma}, P_{\beta \gamma^{\prime}}^{\alpha} r\right)}{\partial g^{\beta \gamma}} .
$$

Then, Equation (39) becomes

$$
\frac{\partial}{\partial \frac{\partial S_{2}^{\lambda}\left(g^{\beta \gamma}, P_{\beta \gamma}^{(\alpha)}, r\right)}{\partial g^{\mu \nu}}} H\left(g^{\mu \nu}, \frac{\partial S_{2}^{\alpha}\left(g^{\beta \gamma}, P_{\beta \gamma}^{(\alpha)}, r\right)}{\partial g^{\mu \nu}}\right)-\widehat{\nabla}_{\lambda} g^{\mu \nu}=0 .
$$

Inspection of Equation (42) shows that this is equivalent in the whole open set $D^{4}$ to the continuum canonical Equation (A9). In fact, in Equation (42), the first term recovers the contribution given by the Poisson bracket on the rhs of Equation (A9). Instead, the second term gives the term on the lhs of 
the same equation, upon identifying $\Pi_{\beta \gamma}^{\alpha}=\frac{\partial S_{2}^{\alpha}\left(g^{\beta \gamma}, P_{\beta \gamma}^{(\alpha)}, r\right)}{\partial g^{\beta \gamma}}$. In addition, the boundary conditions to be satisfied by $\frac{\partial S_{2}^{\alpha}\left(g^{\beta \gamma}, P_{\beta \gamma}^{(\alpha)}, r\right)}{\partial g^{\beta \gamma}}$ and $\Pi_{\beta \gamma}^{\alpha}$ coincide by construction.

In conclusion, the continuum extended Hamilton-Jacobi equation is equivalent to the continuum extended canonical equations. Both equations hold in principle for arbitrary canonical fields $x$, in which the variational 4-tensor $g_{\mu v}$ is still considered different from the background metric tensor $\widehat{g}_{\mu \nu}$.

\section{Search of a Reduced Hamiltonian Theory}

As pointed out above, the extended Hamiltonian theory is characterized by a canonical state $x \equiv\left\{g_{\mu v}, \Pi_{\mu \nu}^{\alpha}\right\}$ in which the Lagrangian coordinate is expressed by the second-order 4-tensor $g_{\mu v}$ while the conjugate momentum remains intrinsically non-unique in the context of the same theory (see Property \#2), being represented by the third-order 4-tensor $\Pi_{\mu v}^{\alpha}$. The discrepancy of tensorial orders between canonical variables is specific to deDonder-Weyl type of manifestly-covariant variational approaches to continuum field dynamics, such as the synchronous principle adopted here for the treatment of the gravitational field in the framework of classical GR. However, the latter feature clashes with the whole formalism of Hamiltonian classical mechanics, both in non-relativistic and relativistic settings, where canonical conjugate variables are naturally born with equal dimension. The same type of problem is then inherited by the extended H-J theory having a 4-tensor Hamilton principal function, which should be expected instead to be expressed by a scalar function.

Two main questions arise at this point. The first one is based on the intrinsic non-uniqueness of the prescription of the canonical momentum $\Pi_{\mu \nu}^{\alpha}$ emerging in the context of the same theory (see Property 2). This suggests the possibility of bringing back the extended theory to a reduced-dimensional representation of the canonical state. This should be achieved in terms of a transformation of the type

$$
x \rightarrow x_{R}
$$

with $x_{R}$ identifying a reduced-dimensional state of the form $x_{R} \equiv\left\{g_{\mu \nu}, \pi_{\mu \nu}\right\}$ and $\pi_{\mu \nu}$ being a suitably-defined reduced canonical momentum represented by an appropriate second-order 4-tensor. In particular, the crucial property which $\pi_{\mu v}$ should exhibit is that (unlike the extended momentum $\Pi_{\mu v}^{\alpha}$ ) of being uniquely determined by the corresponding (reduced) Hamilton equations.

The second issue to be met is again suggested by comparison with the Hamiltonian theory of classical dynamics, where Hamilton equations are expressed as a couple of first-order ordinary differential evolution equations with respect to a suitable evolution parameter, identified with the coordinate time $t$ in non-relativistic mechanics or the proper-time $s$ in relativistic mechanics of classical test particles. Having the Hamilton equations of GR expressed as evolution equations would afford in fact the implementation of standard quantization rules also in the framework of continuum gravitational field theory, such as the Poisson-to-commutator brackets formalism or the Hamilton-Jacobi quantization leading to the Schroedinger-like wave equation. These two requirements are evidently connected to each other, and their simultaneous realization would represent a necessary prerequisite for the consequent establishment of a quantum-gravity theory which is rooted in the Hamiltonian theory of classical GR.

Based on these preliminaries, in this section, the problem of the determination of a reduced continuum Hamiltonian theory for GR is addressed for the Hamiltonian system $\{x, H\}$. This is represented by the reduced classical Hamiltonian structure $\left\{x_{R}, H_{R}\right\}$ which is formed by the appropriate 4-tensor reduced canonical state $x_{R}$ and a suitable 4-scalar Hamiltonian density $H_{R}\left(x_{R}, \widehat{x}_{R}, r, s\right)$. Concerning the notation, here we have included the possibility of a functional dependence of $H_{R}$ on the 4-position $r$ and on the 4-scalar evolution parameter $s$, which at present has a pure symbolic character and will be properly determined in Section 6 . These dependences must be included for generality, as they can arise in principle from the reduction procedure leading 
to Equation (43). In particular, the target requires finding a realization of the variational canonical momentum in such a way that:

(a) In the corresponding reduced canonical state, fields and reduced momenta form a couple of second-order conjugate 4-tensors.

(b) The reduced Hamiltonian theory must yield corresponding suitable reduced continuum Hamilton equations, referred to as reduced GR-Hamilton equations.

(c) The reduced GR-Hamilton equations can be cast in evolution form and warrant the validity of the non-vacuum Einstein field equations.

These requirements are expressed by demanding that the same reduced Hamiltonian equations are realized by the initial-value problem represented by the canonical equations

$$
\left\{\begin{array}{c}
\frac{D g_{\mu v}(s)}{D s}=\frac{\partial H_{R}\left(x_{R}, \widehat{x}_{R}, r, s\right)}{\partial \pi^{\mu v}(s)} \\
\frac{D \pi_{\mu v}(s)}{D s}=-\frac{\partial H_{R}\left(x_{R}, \widehat{x}_{R}, r, s\right)}{\partial g^{\mu v}(s)}
\end{array}\right.
$$

and admit a unique solution when subject to initial conditions of the type

$$
\left\{\begin{array}{l}
g_{\mu v}\left(s_{o}\right) \equiv g_{\mu \nu}^{(o)}\left(s_{o}\right), \\
\pi_{\mu v}\left(s_{o}\right) \equiv \pi_{\mu v}^{(o)}\left(s_{o}\right) .
\end{array}\right.
$$

If such a representation is reached, then the solution of the initial-value problem (44) and (45) generates the Hamiltonian flow

$$
x_{R}\left(s_{0}\right) \rightarrow x_{R}(s),
$$

which is associated with the Hamiltonian structure $\left\{x_{R}, H_{R}\right\}$, where the parameter $s$ must be suitably identified according to the reduction process adopted in Equation (43) (see details below). Hence, here

$$
x_{R}(s) \equiv\left\{g_{\mu v}(r(s)), \pi_{\mu v}(r(s))\right\}
$$

identifies the s-parametrized reduced-dimensional variational canonical state, with $g_{\mu v}$ and $\pi_{\mu v}$ being the corresponding continuum Lagrangian coordinates and the conjugate momenta, $\widehat{x}_{R}(s) \equiv$ $\left\{\widehat{g}_{\mu v}(r(s)), \widehat{\pi}_{\mu v}(r(s)) \equiv 0\right\}$ being the corresponding prescribed state and $H_{R}\left(x_{R}, \widehat{x}_{R}, r, s\right)$ being the variational Hamiltonian 4-scalar density to be suitably determined. Moreover, $\frac{D}{D s}$ is the covariant $s$-derivative defined as the 4-scalar differential operator

$$
\frac{D}{D s} \equiv \frac{\partial}{\partial s}+\Sigma^{\alpha}(s) \hat{\nabla}_{\alpha}
$$

where $\widehat{\nabla}_{\alpha}$ is the covariant derivative evaluated in terms of the prescribed metric tensor $\widehat{g}_{\mu v}$, and $\Sigma^{\alpha}(s)$ is an in principle non-unique suitable "tangent" 4-vector related both to the definition of the evolution parameter $s$ and the reduction procedure yielding the reduced canonical state. The precise identification of $\Sigma^{\alpha}(s)$ is addressed below in Section 6. Finally, the Hamiltonian density $H_{R}\left(x_{R}, \widehat{x}_{R}, r, s\right)$ is represented in terms of the reduced canonical variables as

$$
H_{R}\left(x_{R}, \widehat{x}_{R}, r, s\right) \equiv T_{R}\left(x_{R}, \widehat{x}_{R}\right)+V(g, \widehat{g}, r, s),
$$

where the effective kinetic and potential densities $T_{R}\left(x_{R}, \widehat{x}_{R}\right)$ and $V(g, \widehat{g}, r, s)$ can be taken now respectively of the general form

$$
\left\{\begin{array}{c}
T_{R}\left(x_{R}, \widehat{x}_{R}\right) \equiv \frac{1}{2 \kappa} \pi_{\mu v} \pi^{\mu v}, \\
V(g, \widehat{g}, r, s) \equiv \kappa g^{\mu v} \widehat{R}_{\mu v} h-L_{\Lambda}(g, \widehat{g})-L_{F}(g, \widehat{g}, r, s) .
\end{array}\right.
$$


Given these premises, the construction of the GR-Hamilton Equation (44) follows in analogy with the extended Hamiltonian theory, and in particular they can be derived through a manifestly-covariant synchronous variational approach, in agreement with the deDonder-Weyl approach. The variational functional can be identified now with the real 4-scalar

$$
S_{R}\left(x_{R}, \widehat{x}_{R}\right) \equiv \int d \Omega L_{R}\left(x_{R}, \widehat{x}_{R}, r, s\right),
$$

with $L_{R}\left(x_{R}, \widehat{x}_{R}, r, s\right)$ being the variational Lagrangian density

$$
L_{R}\left(x_{R}, \widehat{x}_{R}, r, s\right) \equiv \pi_{\mu v} \frac{D}{D s} g^{\mu v}-H_{R}\left(x_{R}, \widehat{x}_{R}, r, s\right) .
$$

Thus, $L_{R}\left(x_{R}, \widehat{x}_{R}, r, s\right)$ is identified with the Legendre transform of the corresponding variational Hamiltonian density $H_{R}\left(x_{R}, \widehat{x}_{R}, r, s\right)$ defined by Equation (49). The variational principle associated with the functional $S_{R}\left(x_{R}, \widehat{x}_{R}\right)$ is prescribed in terms of the synchronous-variation operator $\delta$ (i.e., identified with the Frechet derivative according to Ref. [10]), i.e., by means of the synchronous variational principle

$$
\delta S_{R}\left(x_{R}, \widehat{x}_{R}\right)=0
$$

obtained keeping constant both the prescribed state $\widehat{x}_{R}$ and the 4 -scalar volume element $d \Omega$. This yields the 4-tensor Euler-Lagrange equations cast in symbolic form

$$
\left\{\begin{array}{l}
\frac{\delta S_{R}\left(x_{R}, \widehat{x}_{R}\right)}{\delta g^{\mu v}}=0, \\
\frac{\delta S_{R}\left(x_{R}, \widehat{x}_{R}\right)}{\delta \pi_{\mu v}}=0,
\end{array}\right.
$$

which are manifestly equivalent to the Hamilton Equation (44). These equations can be written in the equivalent Poisson-bracket representation as

$$
\frac{D}{D s} x_{R}(s)=\left[x_{R}, H_{R}\left(x_{R}, \widehat{x}_{R}, r, s\right)\right]_{\left(x_{R}\right)},
$$

with $[,]_{\left(x_{R}\right)}$ denoting the Poisson bracket evaluated with respect to the canonical variables $x_{R}$, namely

$$
\left[x_{R}, H_{R}\left(x_{R}, \widehat{x}_{R}, r, s\right)\right]_{\left(x_{R}\right)}=\frac{\partial x_{R}}{\partial g^{\mu v}} \frac{\partial H_{R}}{\partial \pi_{\mu v}}-\frac{\partial x_{R}}{\partial \pi_{\mu v}} \frac{\partial H_{R}}{\partial g^{\mu v}} .
$$

The connection of Equation (55) with the Einstein field equations can then be shown to follow by requiring validity of the initial conditions

$$
\left\{\begin{array}{c}
g_{\mu v}\left(s_{o}\right) \equiv \widehat{g}_{\mu v}\left(s_{o}\right), \\
\pi_{\mu v}\left(s_{o}\right) \equiv \widehat{\pi}_{\mu v}\left(s_{o}\right)=0,
\end{array}\right.
$$

together with the requirement $\widehat{\pi}_{\mu v}(s)=0$ holding for all $s \in I$.

\section{Reduced Hamilton-Jacobi Theory}

In this section, we analyze the requirements for the establishment of a reduced Hamilton-Jacobi theory of classical GR that can be derived from the extended Hamilton-Jacobi formulation presented in Section 3. This consists of the realization of a Hamilton-Jacobi equation in which the Hamilton principal function is expressed as a 4 -scalar $S$ obtained through a suitable reduction procedure from the 4 -vector principal function $S_{2}^{\alpha}$. The sought theory must be proved to be equivalent both to the extended Hamilton-Jacobi theory as well as to the extended and reduced Hamiltonian theories formulated above.

The derivation presented here follows from the reduced Hamiltonian theory and the representation of the GR-Hamilton equations as dynamical evolution equations. This feature in 
fact is appropriate for the development of the theory of canonical transformations in close analogy with classical Hamiltonian mechanics. More precisely, in distinction with the extended Hamilton-Jacobi continuum theory, the corresponding reduced theory should describe a dynamical flow connecting a generic phase-space state $x_{R} \equiv\left\{g_{\mu v}, \pi_{\mu v}\right\}$ with a suitable initial phase-space state $X_{R} \equiv\left\{G_{\mu v}, P_{\mu v}\right\}$ characterized by constant coordinate fields and momenta. This includes as a special case the configuration in which coordinate fields and momenta are identically-vanishing, i.e., stationary with respect to the evolution parameter $s$. It is then expected that the reduced Hamilton-Jacobi theory follows from constructing a symplectic canonical transformation associated with a mixed-variable generating function of type $S\left(g \beta \gamma, P_{\mu v}, \widehat{x}_{R}, r, s\right)$.

Accordingly, the transformed canonical state $X_{R}$ must satisfy the constraint equations

$$
\begin{aligned}
\frac{D}{D s} P_{\mu v}\left(s_{0}\right) & =0, \\
\frac{D}{D s} G^{\mu v}\left(s_{0}\right) & =0,
\end{aligned}
$$

which imply the Hamilton equations

$$
\begin{aligned}
0 & =\left[P_{\mu v}, K_{R}\left(X_{R}, \widehat{X}_{R}, r, s\right)\right]_{\left(X_{R}\right)^{\prime}} \\
0 & =\left[G^{\mu v}, K_{R}\left(X_{R}, \widehat{X}_{R}, r, s\right)\right]_{\left(X_{R}\right)^{\prime}}
\end{aligned}
$$

where $K_{R}\left(X_{R}, \widehat{X}_{R}, r, s\right)$ is the transformed Hamiltonian given by

$$
K_{R}\left(X_{R}, \widehat{X}_{R}, r\right)=H_{R}\left(x_{R}, \widehat{x}_{R}, r, s\right)+\frac{\partial}{\partial s} S\left(g^{\beta \gamma}, P_{\mu v}, \widehat{x}_{R}, r, s\right) .
$$

Thanks to Equations (60) and (61), the transformed Hamiltonian is necessarily independent of $X_{R}$. As a consequence, $K_{R}$ identifies an arbitrary gauge function, i.e., it must be $K_{R}=K_{R}\left(\widehat{x}_{R}, r\right)$, which can always be set equal to zero $\left(K_{R}=0\right)$. On the other hand, canonical transformation theory requires that it must be

$$
\begin{aligned}
\pi_{\iota \xi} & =\frac{\partial S\left(g^{\beta \gamma}, P_{\mu v}, \widehat{x}_{R}, r, s\right)}{\partial g^{\prime \xi}} \\
G^{\iota \xi} & =\frac{\partial S\left(g^{\beta \gamma}, P_{\mu v}, \widehat{x}_{R}, r, s\right)}{\partial P_{\iota \xi}} .
\end{aligned}
$$

It follows that Equation (62) delivers

$$
H_{R}\left(g^{\beta \gamma}, \frac{\partial S\left(g^{\beta \gamma}, P_{\mu \nu}, \widehat{x}_{R}, s\right)}{\partial g^{\prime \zeta}}, \widehat{x}_{R}, r, s\right)+\frac{\partial}{\partial s} S\left(g^{\beta \gamma}, P_{\mu v}, \widehat{x}_{R}, r, s\right)=0,
$$

which realizes the reduced GR Hamilton-Jacobi equation for the mixed-variable generating function $S\left(g^{\beta \gamma}, P_{\mu v}, \widehat{x}_{R}, r, s\right)$. Due to its similarity with the customary Hamilton-Jacobi equation well-known in Hamiltonian classical dynamics, in the following, $S$ will be referred to as the reduced GR-Hamilton principal function. The canonical transformations generated by $S\left(g^{\beta \gamma}, P_{\mu v}, \widehat{x}_{R}, r, s\right)$ are then obtained by the set of Equations (63)-(65). The inverse canonical transformation $X_{R} \rightarrow x_{R}$ locally exists provided the invertibility condition on the Hessian determinant det $\left|\left[\frac{\partial^{2} S\left(g^{\beta \gamma}, P_{\mu v}, \widehat{x}_{R}, r, s\right)}{\partial g^{\rho \sigma \partial} \partial P_{\iota \xi}}\right]_{X_{R}=\widehat{x}_{R}}\right| \neq 0$ is met. Under such a condition, the direct canonical Equation (64) determines $g^{\beta \gamma}$ as an implicit function of the form $g^{\beta \gamma}=g^{\beta \gamma}\left(G^{\beta \gamma}, P_{\mu v}, \widehat{x}_{R}, r, s\right)$. 
We now proceed proving the equivalence between reduced GR-Hamilton and GR-Hamilton-Jacobi equations, in analogy with the relationship holding for the corresponding extended formulations. To this aim, the compact notation $S\left(g, P, \widehat{x}_{R}, r, s\right)$ is adopted below. In particular, it follows that the GR-Hamilton-Jacobi Equation (65) subject to the constraint (63) is equivalent to the set of GR-Hamilton equations expressed in terms of the initial canonical variables, as given by Equation (44). To start with, we evaluate first the partial derivative of Equation (65) with respect to $g^{i k}$, keeping both $\frac{\partial S\left(g, P, \widehat{x}_{R}, r, s\right)}{\partial g^{i / \xi}}$ and $r^{\mu}$ constant. This gives

$$
\frac{\partial}{\partial g^{i k}} H_{R}\left(g^{\beta \gamma}, \frac{\partial S\left(g, P, \widehat{x}_{R}, s\right)}{\partial g^{\prime \zeta}}, \widehat{x}_{R}, r, s\right)+\frac{\partial}{\partial s}\left[\frac{\partial}{\partial g i k} S\left(g, P, \widehat{x}_{R}, r, s\right)\right]_{(g, P)}=0 .
$$

Then, we evaluate in a similar manner the partial derivative with respect to $\frac{\partial S\left(g, P, \widehat{x}_{R}, s\right)}{\partial g^{i k}}$, keeping $g^{\mu \nu}$ and $r^{\mu}$ constant. This gives

$$
\frac{\partial}{\partial \frac{\partial S\left(g, P, \widehat{x}_{R}, r, s\right)}{\partial g^{i k}}} H_{R}\left(g^{\beta \gamma}, \frac{\partial S\left(g, P, \widehat{x}_{R}, s\right)}{\partial g^{\iota \xi}}, \widehat{x}_{R}, r, s\right)+\left[\frac{\partial}{\partial \frac{\partial S\left(g, P, \widehat{x}_{R}, r, s\right)}{\partial g^{i k}}} \frac{\partial}{\partial s} S\left(g, P, \widehat{x}_{R}, r, s\right)\right]_{(g, P)}=0 .
$$

With the identification $\pi_{\iota \xi}=\frac{\partial S\left(g, P, \widehat{x}_{R}, S\right)}{\partial g^{\prime \tau},}$ provided by Equation (63), it follows that Equation (66) becomes

$$
\frac{\partial}{\partial g^{i k}} H_{R}\left(g^{\beta \gamma}, \pi_{\iota \xi}, \widehat{x}_{R}, r\right)+\frac{D}{D s} \pi_{i k}=0,
$$

which coincides with the second Hamilton equation in (44). To prove also the validity of the Hamilton equation for $g^{\beta \gamma}$, we first invoke the following identity

$$
\begin{gathered}
{\left[\frac{\partial}{\partial \frac{\partial S\left(g, P, \widehat{x}_{R}, s\right)}{\partial g^{i k}}} \frac{\partial}{\partial s} S\left(g, P, \widehat{x}_{R}, s\right)\right]_{(g, P)}=\frac{\partial}{\partial \frac{\partial S\left(g, P, \widehat{x}_{R}, s\right)}{\partial g^{i k}} \frac{\partial}{\partial s} S\left(g, P, \widehat{x}_{R}, s\right)}} \\
-\frac{D}{D s} g^{\beta \gamma} \frac{\partial}{\partial \frac{\partial S\left(g, P, \widehat{x}_{R}, s\right)}{\partial g^{i k}}} \frac{\partial S\left(g, P, \widehat{x}_{R}, s\right)}{\partial g \beta \gamma},
\end{gathered}
$$

where

$$
\frac{\partial}{\partial \frac{\partial S\left(g, P, \widehat{x}_{R}, r, s\right)}{\partial g^{i k}}} \frac{\partial S\left(g, P, \widehat{x}_{R}, r, s\right)}{\partial g \beta \gamma}=\delta_{\beta}^{i} \delta_{\gamma}^{k}
$$

The first term on the rhs of Equation (69) vanishes identically because $\frac{\partial}{\partial s} S\left(g, P, \widehat{x}_{R}, r, s\right)$ must be considered as independent of $\pi_{i k}$. Therefore, Equation (67) gives

$$
\frac{\partial}{\partial \pi_{i k}} H_{R}\left(g^{\beta \gamma}, \pi_{\iota} \tilde{s}, \widehat{x}_{R}, r, s\right)-\frac{D}{D s} g^{i k}=0,
$$

which coincides with the Hamilton equation for $g^{i k}$ and gives also the relationship of the generalized velocity $\frac{D}{D s} g^{i k}$ with the canonical momentum. This proves the equivalence between the reduced GR-Hamilton-Jacobi and GR-Hamilton equations, both expressed in manifestly-covariant form.

This conclusion recovers the relationship between Hamilton and Hamilton-Jacobi equations holding in Hamiltonian Classical Mechanics for discrete dynamical systems. The connection is established also in the present case for the continuum gravitational field thanks to the manifestly-covariant representation of the Hamiltonian and Hamilton-Jacobi equations as dynamical evolution equations with respect to the dynamical parameter $s$, to be defined below. The physical interpretation which follows concerns the meaning of the Hamilton-Jacobi theory in providing a wave mechanics description of the continuum Hamiltonian dynamics. This arises also in the present context 
by comparing the mathematical structure of the Hamilton-Jacobi Equation (65) with the well-known eikonal equation of geometrical optics. In fact, Equation (65) contains the square of the derivative $\frac{\partial S\left(g^{\beta \gamma}, P_{\mu v}, \widehat{x}_{R}, r, s\right)}{\partial g^{l \zeta}}$, so that the Hamilton principal function $S\left(g^{\beta \gamma}, P_{\mu v}, \widehat{x}_{R}, r, s\right)$ is associated with the eikonal (i.e., the phase of the wave), while the remaining contributions due to the geometrical and physical properties of the curved space-time formally play the role of a non-uniform index of refraction in geometrical optics [32]. The outcome pointed out here proves that the dynamics of the field $g_{\mu v}(s)$ in the domain of variational fields where the reduced Hamiltonian structure is defined and the reduced Hamilton-Jacobi theory (65) applies must be characterized by a wave-like behavior and can therefore be given a geometrical optics interpretation. This feature is fundamental for the establishment of the corresponding manifestly-covariant quantum theory of the gravitational field.

\section{The Projection Operator}

In this section, we set up the mathematical method that is used to generate reduced Hamilton and Hamilton-Jacobi theories starting from the corresponding extended formulations. This is accomplished by introducing the projection operator $\Sigma$, i.e., by definition an operator that acting on an arbitrary tensor function of the extended canonical state $x$ is such that $\Sigma \Sigma=\frac{1}{\Gamma}$, with 1 being the unity operator and $\Gamma$ a real constant. Thus, in the case in which $\Gamma=1, \Sigma$ identifies a so-called normalized projection operator. This should be defined so that, in particular, it generates the reduced canonical state $x_{R}$, namely

$$
x_{R}=\Sigma[x] .
$$

Based on the prescription of $x$ and $x_{R}$ given above, this requires accordingly that when acting on the extended or reduced canonical momenta $\Pi_{\mu v}^{\alpha}$ and $\pi_{\mu v}$ it should yield the other momentum, i.e., respectively

$$
\left\{\begin{array}{c}
\pi_{\mu v}=\Sigma\left[\Pi_{\mu v}^{\alpha}\right], \\
\Sigma \pi_{\mu v}=\Sigma \Sigma\left[\Pi_{\mu v}^{\alpha}\right]=(\Gamma)^{-1} \Pi_{\mu v}^{\alpha} .
\end{array}\right.
$$

The simplest realization of Equation (73) is thus obtained by identifying the projection operator with the 4 -vector $\Sigma^{\alpha}$, namely such that

$$
\left\{\begin{array}{c}
\Sigma \equiv \Sigma^{\alpha} \\
\Sigma \Sigma \equiv \Sigma^{\alpha} \Sigma_{\alpha}=(\Gamma)^{-1}
\end{array}\right.
$$

which implies the validity of the tensor products

$$
\left\{\begin{array}{c}
\Sigma_{\alpha} \Pi_{\mu v}^{\alpha}=\pi_{\mu v} \\
\Sigma^{\alpha} \pi_{\mu v}=(\Gamma)^{-1} \Pi_{\mu v}^{\alpha} .
\end{array}\right.
$$

The physical meaning of the two relationships is immediate: $\pi_{\mu v}$ is obtained, up to a factor, by projecting the 3 rd-order tensor $\Pi_{\mu v}^{\alpha}$ along the 4 -vector $\Sigma_{\alpha}$, which saturates one tensorial index. Similarly, $\Pi_{\mu \nu}^{\alpha}$ is obtained up to a suitable factor by multiplying with $\Sigma^{\alpha}$ the reduced canonical momentum. In an analogous way, one can introduce for the Hamilton principal functions the relationship

$$
S=\Sigma_{\alpha} S^{\alpha},
$$

with $S$ and $S^{\alpha}$ representing respectively the corresponding reduced and extended functions. In order to achieve the reduced canonical formulation reported above (see previous sections) and consistent with the prescriptions (75), the identification of the projection operator must satisfy the following requirements: 
(A) The Definitions (75) and (76) must be invertible, namely it must be equally possible to express $\Pi_{\mu v}^{\alpha}$ as

$$
\Pi_{\mu v}^{\alpha}=\frac{1}{\Gamma} \Sigma^{\alpha} \pi_{\mu v}
$$

and $S^{\alpha}$ as

$$
S^{\alpha}=\frac{1}{\Gamma} \Sigma^{\alpha} S,
$$

where $\Gamma \equiv \Sigma^{\alpha} \Sigma_{\alpha}$ is the magnitude of the 4 -vector $\Sigma^{\alpha}$. Therefore, it must be $\Gamma \neq 0$ necessarily.

(B) The 4-vector $\Sigma^{\alpha}$ cannot be identified with a differential operator, otherwise it would alter the differential order of both Hamilton and Hamilton-Jacobi equations. As a consequence, it follows that in particular it must be $\Sigma^{\alpha} \neq \nabla^{\alpha}$.

(C) The 4-vector $\Sigma^{\alpha}$ must commute with the differential operators $\widehat{\nabla}^{\alpha}$ and $\left(\frac{\partial}{\partial g_{\mu \nu}}, \frac{\partial}{\partial g^{\mu v}}\right)$, in order to afford the evolution representation of the canonical equations and the definition of covariant derivative according to Equation (48).

(D) The definition of $\Sigma^{\alpha}$ must hold for a generic background space-time $\widehat{g}_{\mu v}$, and in particular also in the limit of flat space-time for which $\widehat{g}_{\mu \nu} \equiv \eta_{\mu v}$, being $\eta_{\mu v}$ the Minkowski metric tensor. This limit in fact is physically relevant, since the quantum theory of gravitational field stemming from the canonical formulation of GR must apply also in such a case.

(E) The definition of $\Sigma^{\alpha}$ must be such to warrant that the reduced Hamiltonian structure $\left\{x_{R}, H_{R}\right\}$ and the form of reduced Hamiltonian equations are preserved, included the quadratic dependence on the canonical momenta.

(F) Since the projector operator acts only on the kinetic term of the Hamiltonian function and leaves unaffected the potential term, the 4 -vector $\Sigma^{\alpha}$ must be dimensionless, so that the physical dimensions of the extended and reduced momenta remain the same and are dimensionally homogeneous with the potential term of the Hamiltonian.

(G) Finally, the reduced canonical state $x_{R}=\Sigma[x]$ and in particular the corresponding reduced canonical momentum $\pi_{\mu v}=\Sigma_{\alpha} \Pi_{\mu v}^{\alpha}$ should be uniquely determined by the initial-value problem (44) and (45).

The conditions $(A)-(G)$ require that the explicit representation of $\Sigma^{\alpha}$ must be defined with respect to quantities of the background space-time only. Although in principle the choice of $\Sigma^{\alpha}$ could span a wide range of possibilities, after careful analysis, one can conclude that there are only two candidates that could fall in this category with a precise physical meaning.

The first one is met by identifying $\Sigma^{\alpha}$ with the wave 4-vector $k^{\alpha}$ associated with light-ray geodesic trajectories. By definition, this satisfies the conditions

$$
\begin{aligned}
k^{\alpha} k_{\alpha} & =0, \\
D k^{\alpha} & =0,
\end{aligned}
$$

where here $D$ stands for the customary coordinate covariant derivative operator of the space-time $\widehat{g}_{\mu \nu}$ (see Ref. [14]). If acceptable, this choice for the projection operator would correspond to the physical map of the canonical momenta and their dynamics along the space-time background direction determined by wavefronts of luminal geodesic trajectories. However, it is easy to verify that this solution is not appropriate, as the choice of the wave-vector $k^{\alpha}$ satisfies only the requirements (B)-(D) stated above. Instead, the criterion (A) is violated since in such a case $\Gamma=0$, so that the projection transformation is not invertible, while similarly point $(\mathrm{E})$ is violated because projecting the momenta along $k^{\alpha}$ makes the kinetic term of the Hamiltonian function vanish identically. In fact, invoking the commutation property of point (C), one has that $\Pi_{\mu v}^{\alpha} \Pi_{\alpha}^{\mu v}=\left(k^{\alpha} k_{\alpha}\right) \pi_{\mu v} \pi^{\mu v}=0$, which implies the whole Hamiltonian structure of GR to break down and the theory to collapse to a hypersurface where only the potential contribution survives, therefore to a state without dynamics. In conclusion, this type of projection must be excluded. 
The second choice corresponds instead to setting $\Sigma^{\alpha}=t^{\alpha}$, where $t^{\alpha}$ is the tangent 4 -vector belonging to sub-luminal geodesics defined in the background space-time $\widehat{g}_{\mu \nu}$ with arc length prescribed by the differential equation

$$
d s^{2}=\widehat{g}_{\mu \nu} d r^{\mu} d r^{v}
$$

Here, $d r^{\mu}$ is the tangent displacement with respect to the same geodesics, so that by construction it follows that $t^{\alpha} \equiv \frac{d r^{\alpha}}{d s}$ is the corresponding tangent 4-velocity, with the parameter $s$ identifying the invariant proper-time associated with the same geodetic curve. Thus, by definition the tangent 4-vector satisfies the conditions

$$
\begin{aligned}
t^{\alpha} t_{\alpha} & =1, \\
D t^{\alpha} & =0,
\end{aligned}
$$

so that in this case $\Gamma=1$ identically and condition (A) is verified. As a consequence, the relationships between extended and reduced momenta become now simply $\Pi_{\mu \nu}^{\alpha}=t^{\alpha} \pi_{\mu v}$ and $S^{\alpha}=t^{\alpha} S$. An additional important feature of this solution is that $t^{\alpha}$ is dimensionless, as required by condition $(\mathrm{F})$, a feature which warrants validity of the Hamiltonian theory. The remaining conditions (B)-(E) and (G) listed above can be shown to be satisfied as well, so that the identification $\Sigma^{\alpha}=t^{\alpha}$ remains the only acceptable one. From the physical point of view, this corresponds to the projection of the Hamiltonian dynamics along a sub-luminal direction accessible to massive test particles (i.e., the observers). This choice, in addition, provides a unique identification also for the evolution parameter to be used in the reduced canonical equations, which is represented by the proper-time $s$ measured along the same curve.

After the identification of the projection operator $\Sigma^{\alpha}=t^{\alpha}$ and the evolution parameter with the proper-time $s$ associated with sub-luminal geodesic trajectories, we can address in more detail the representation of the canonical equations in evolution form. As anticipated above, this represents a requirement for the construction of a corresponding manifestly-covariant Hamilton-Jacobi theory of GR from the Hamiltonian theory, and its subsequent quantization. In particular, we introduce here the notion of Lagrangian path (LP) [31] and the related concept of Lagrangian path parametrization of Hamilton equations [22].

We first express the functional dependence of the tangent 4 -vector $t^{\alpha}$, which is of the type $t^{\alpha}(\widehat{g}(s), r)$. Then, Equations (82) and (83) can be written as

$$
\left\{\begin{array}{c}
t^{\alpha}(\widehat{g}(s), r) \widehat{\nabla}_{\alpha} t^{\gamma}(\widehat{g}(s), r)=0, \\
\widehat{g}_{\mu v}(s) t^{\mu}(\widehat{g}(s), r) t^{v}(\widehat{g}(s), r)=1,
\end{array}\right.
$$

so that by construction $t^{\alpha}(\widehat{g}(s), r)$ is tangent to an arbitrary sub-luminal geodetics belonging to an arbitrary 4-position $r \equiv\left\{r^{\mu}\right\}$ of the space-time $\left(\mathbf{Q}^{4}, \widehat{g}(s)\right)$ [14]. Then, the LP is identified with the geodetic curve

$$
\left\{r^{\mu}(s)\right\} \equiv\left\{r^{\mu}(s) \mid \forall s \in \mathbb{R}, r^{\mu}\left(s_{o}\right)=r_{o}^{\mu}\right\},
$$

which is a solution of the initial-value problem

$$
\left\{\begin{array}{c}
\frac{d r^{\mu}(s)}{d s}=t^{\mu}(s), \\
r^{\mu}\left(s_{o}\right)=r_{o}^{\mu} .
\end{array}\right.
$$

Here, the 4-scalar proper-time $s$ is defined along the same curve $\left\{r^{\mu}(s)\right\}$ so that $d s^{2}=$ $\widehat{g}_{\mu v}(r) d r^{\mu}(s) d r^{v}(s)$. Furthermore, $t^{\mu}(s)$ identifies the LP-parametrized 4-vector $t^{\mu}(s) \equiv$ $t^{\mu}(\widehat{g}(r(s)), r(s))$. In Equation (86), $\frac{d}{d s} \equiv \frac{\partial}{\partial s}$ identifies the ordinary derivative with respect to $s$. According to the notation introduced in Ref. [19], we distinguish between implicit and explicit 
$s$-dependences, whereby the implicit ones are the dependences on the proper time $s$ appearing in the variational fields through the LP parametrization of the fields, while the explicit ones are the proper-time dependences which enter either explicitly on $s$ itself or through the dependence on $r(s) \equiv\left\{r^{\mu}(s)\right\}$.

The parametrization obtained replacing everywhere, in all the relevant tensor fields, $r \equiv\left\{r^{\mu}\right\}$ with $r(s) \equiv\left\{r^{\mu}(s)\right\}$, namely obtained identifying

$$
\left\{\begin{aligned}
g^{\mu v}(s) & \equiv g^{\mu v}(r(s)), \\
\pi^{\mu \nu}(s) & \equiv \pi^{\mu v}(r(s)), \\
\widehat{x}(r) & \equiv \widehat{x}(r(s)),
\end{aligned}\right.
$$

yields for the Hamiltonian density $H_{R}$ the so-called LP-parametrization. In terms of this formalism, the reduced Hamilton Equation (44) and correspondingly the reduced Hamilton-Jacobi Equation (65) can in turn be represented, where for greater generality the reduced Hamiltonian function is taken of the form $H_{R}(s) \equiv H_{R}\left(x_{R}(s), \widehat{x}_{R}(s), r(s), s\right)$, i.e., including also a possible explicit dependence in terms of the proper time $s$.

\section{Hamilton-Jacobi Waves}

In the previous section, we have shown that, given the set of requirements for the choice of the projection operator, of the two available options $\Sigma^{\alpha}=k^{\alpha}$ and $\Sigma^{\alpha}=t^{\alpha}$, only the latter is physically-admissible as a single-direction representation of the conjugate momenta. In this section, we explore in more detail the issue, starting directly from the point of view of the extended and reduced Hamilton-Jacobi theory, in order to shed further light on the wave theory underlying the canonical formulation of classical GR and its conceptual consequences on the corresponding quantum formulation.

In fact, although $\Sigma^{\alpha}=k^{\alpha}$ remains excluded tout court as projection operator, there still remains the possibility of considering a more complex solution expressed as a linear combination between $k^{\alpha}$ and $t^{\alpha}$, namely obtained decomposing the extended Hamilton principal function according to the following type of representation:

$$
S_{2}^{\alpha}=t^{\alpha} S_{t}+k^{\alpha} S_{k}
$$

where $S_{t}$ and $S_{k}$ are 4-scalars and the sub-scripts identify their respective 4-vector projection direction. Equation (88) is valid under the assumption that it must be $S_{t} \neq 0$, while $S_{k}$ could also vanish in principle, and if this happens the solution obtained in Section 6 is recovered. We notice however that the two functions $S_{t}$ and $S_{k}$ have different physical dimensions, since $t^{\alpha}$ is a tangent 4-vector, while $k^{\alpha}$ is a wave 4 -vector. From the physical point of view, the first term proportional to $t^{\alpha}$ is associated with sub-luminal geodetics and massive-particle waves, while $k^{\alpha}$ with luminal geodetics and corresponding massless waves. We notice that the previous combination still satisfies the requirements set in Section 6 , thanks to the dependence on the term $t^{\alpha} S_{t}$. On the other hand, the same representation introduces the two 4-scalar functions $S_{t}$ and $S_{k}$, which cannot be both solved by the Hamilton-Jacobi equation, as this is a 4 -scalar equation. This requires therefore to determine $S_{k}$ separately. To this aim, we substitute the representation (88) in the extended Hamilton-Jacobi Equation (35) and we study the different differential contributions:

$$
\begin{aligned}
\hat{\nabla}_{\alpha} S_{2}^{\alpha} & \longrightarrow t^{\alpha} \hat{\nabla}_{\alpha} S_{t}+k^{\alpha} \hat{\nabla}_{\alpha} S_{k}, \\
\frac{\partial S_{2}^{\alpha}}{\partial g_{\mu v}} & =t^{\alpha} \frac{\partial S_{t}}{\partial g_{\mu v}}+k^{\alpha} \frac{\partial S_{k}}{\partial g_{\mu v}},
\end{aligned}
$$

from which one has

$$
\begin{aligned}
\frac{\partial S_{2}^{\alpha}}{\partial g_{\mu v}} \frac{\partial S_{2 \alpha}}{\partial g^{\mu v}} & \longrightarrow t^{\alpha} t_{\alpha} \frac{\partial S_{t}}{\partial g_{\mu v}} \frac{\partial S_{t}}{\partial g^{\mu v}}+k^{\alpha} k_{\alpha} \frac{\partial S_{k}}{\partial g_{\mu k}} \frac{\partial S_{k}}{\partial g^{\mu v}}+2 t^{\alpha} k_{\alpha} \frac{\partial S_{t}}{\partial g_{\mu v}} \frac{\partial S_{k}}{\partial g^{k v}} \\
& =\frac{\partial S_{t}}{\partial g_{\mu \nu}} \frac{\partial S_{t}}{\partial g^{\mu v}}+2 t^{\alpha} k_{\alpha} \frac{\partial S_{t}}{\partial g_{\mu \nu}} \frac{\partial S_{k}}{\partial g^{k v}}
\end{aligned}
$$


where in the last line we have used the properties $t^{\alpha} t_{\alpha}=1$ and $k^{\alpha} k_{\alpha}=0$.

Since the contribution $k^{\alpha} S_{k}$ in Equation (88) describes massless waves, we impose that the representation of the 4 -scalar $S_{k}$ admits a variable separation of the type

$$
S_{k}=S_{o} f\left(k_{\mu} r^{\mu}\right),
$$

where the dependence on the 4-position $r^{\mu}$ only enters through the function $f$ in terms of the 4-scalar combination $k_{\mu} r^{\mu}$ associated with the wave 4 -vector $k^{\alpha}$. A possible choice is through the wave

$$
S_{k}=S_{o} e^{i k_{\mu} r^{\mu}}
$$

where it is understood that only the real component matters. Instead, in Equation (92), the 4-scalar $S_{o}$ is either constant or dependent on $g_{\mu \nu}$ only. Thanks to this representation in Equation (89), we obtain that $k^{\alpha} \widehat{\nabla}_{\alpha} S_{k}=0$ identically, since the derivative generates again a term proportional to $k^{\alpha} k_{\alpha}=0$, so that the contribution of $S_{k}$ in the linear differential term of the Hamilton-Jacobi equation is null. The only surviving contribution containing $S_{k}$ is in the mixed quadratic term carried by the Hamiltonian function, which according to Equation (91) becomes

$$
2 t^{\alpha} k_{\alpha} \frac{\partial S_{t}}{\partial g_{\mu v}} \frac{\partial S_{k}}{\partial g^{\mu \nu}}=2 t^{\alpha} k_{\alpha} f\left(k_{\mu} r^{\mu}\right) \frac{\partial S_{t}}{\partial g_{\mu \nu}} \frac{\partial S_{o}}{\partial g^{\mu \nu}} .
$$

A trivial realization is obtained when $\frac{\partial S_{0}}{\partial g^{\mu \nu}} \equiv 0$, namely $S_{o}=$ const. In this case, the massless term $k^{\alpha} S_{k}$ in Equation (88) becomes completely determined while its contribution to the Hamilton-Jacobi equation obviously vanishes by construction. We remark, however, that it is possible to prove that the choice $S_{o}=$ const . remains the only admissible one. For completeness, the proof of this property is reported in Appendix B.

In summary, a more general choice for the representation of the Hamilton principal function is according to the type provided by Equation (88) as follows:

$$
S_{2}^{\alpha}=t^{\alpha} S_{t}+k^{\alpha} S_{o} e^{i k_{\mu} r^{\mu}}
$$

where we set $S_{o}=$ const. In this way, the classical solution presented in Section 6 as well as the classical and quantum solutions investigated in Refs. $[19,20]$ for the choice $t^{\alpha} S_{t}$ remain unaffected. The conclusion in fact is the following: the extended Hamilton-Jacobi equation admits in principle a representation of the solution for the Hamilton principal function which is more general than the one considered so far and proportional only to the massive term $t^{\alpha} S_{t}$, which is of the type (95). This choice however leaves unaffected both the classical and quantum solutions previously investigated elsewhere and corresponding to the existence of massive gravitons. The new term added here in Equation (95) has a tensorial direction determined by the wave 4-vector $k^{\alpha}$ and it describes the classical contribution of luminal waves (i.e., massless gravitons in the language of quantum theory). For this reason, the functional dependence on the coordinate 4-position $r^{\alpha}$ has been chosen to be the wave-like one. The solution expressed by the linear combination in Equation (95) is therefore an admissible solution, which generalizes the particular case $S_{2}^{\alpha}=t^{\alpha} S_{t}$. As a remarkable conclusion, however, we stress that the same combination of massive and massless wave contributions to $S_{2}^{\alpha}$ only matters for the classical Hamilton-Jacobi theory, since after substitution the massless term cancels out and only the massive term survives and is transferred to the realm of corresponding quantum theory. The quantum dynamics can only treat wave-like solutions corresponding to massive gravitons. Therefore, according to the present theory, the reduced representation can include both massive and massless classical wave contributions, but the massless waves have only a classical connotation and they cannot pertain to the quantum gravity theory built upon the GR canonical formalism. 


\section{Conclusions}

The manifestly-covariant approach to the canonical formulation of General Relativity provides a new self-consistent route to Quantum Gravity and a promising scenario for the investigation of the mathematical properties of an unconstrained Hamiltonian theory of the gravitational field in which canonical variables are independent, with the canonical momentum being characterized, at the same time, by a reduced dimension with respect to the standard deDonder-Weyl approach. Indeed, it is generally agreed that a theory of this type-besides remaining in agreement with the fundamental principles of the Einstein theory of GR-should also for consistency recover the Einstein field equations, possibly without recurring to a semiclassical limit of the theory. In this investigation, this refers specifically to the realization of the corresponding classical formulation, i.e., the covariant classical gravity theory (CCG-theory).

More precisely, in this paper, a number of results have been reported.

These include, first, the analysis given in Section 2 of the deDonder-Weyl synchronous-variational approach which is set at the basis of CCG-theory. In particular, for this purpose, here the connection has been established between the extended Hamiltonian equations generated in this way with the customary set of Einstein field equations (see Property 1). However, a non-uniqueness feature (of the solutions of the same equations) has been pointed out which characterizes the extended mixed-order canonical state $x$. Indeed, for arbitrary boundary conditions, the extended canonical momentum $\Pi \equiv\left\{\Pi_{\mu v}^{\alpha}\right\}$ is found to remain actually undetermined (Property 2). Despite these conclusions, the corresponding canonical transformations and extended Hamilton-Jacobi theories can still be formally established and have been reported here for the first time (see Section 3).

Second, the construction of reduced Hamiltonian and corresponding Hamilton-Jacobi theories has been investigated (see Sections 4 and 5, respectively). Motivated by the intrinsic non-uniqueness of the extended canonical momentum, this has been achieved by means of the introduction of a suitable reduced-dimensional canonical state $x_{R}$ with the corresponding momentum $\pi \equiv\left\{\pi_{\mu v}\right\}$ to be determined by the corresponding (reduced) Hamilton equations.

Third, a formal projection-operator has been established (Section 6), which enables the determination of the explicit connection between extended and reduced Hamiltonian theories.

The crucial feature displayed here is that all the relevant equations, in particular both in the extended and reduced Hamiltonian representations, are frame-independent and hence do not require the choice of a particular subset of coordinate-systems nor the introduction of any sort of space-time foliation. The basic implication is that the compact tensorial formalism which characterizes manifestly-covariant theories like GR remains preserved and represents by itself an asset able to gain insight into the same canonical theory. More precisely, the manifestly-covariant representation allows for displaying the symmetry relationships existing between the classical Einstein field equations of General Relativity and its classical variational formulation, which is realized in terms of corresponding Lagrangian, Hamiltonian and Hamilton-Jacobi theories. The equivalence and mutual implications of these three levels of representations have been detailed in this paper. The primary interest of the work, besides the theoretical foundations of General Relativity and the completeness of its conceptual framework, lies in the possibility of providing an appropriate setting for the development of a manifestly-covariant quantum gravity theory. This step can in principle be equivalently implemented either in terms of a canonical Poisson-bracket formulation or passing through the Hamilton-Jacobi quantization leading to the derivation of an appropriate Schroedinger-like quantum wave equation for the gravitational field.

The main conclusions drawn here are relevant for their consequences also on covariant quantum gravity theory. In fact, although the theory developed here describes consistently both luminal and subluminal wave phenomenology of the gravitational field, only sub-luminal solutions pertain the subsequent quantization. In particular, the manifestly-covariant Hamilton-Jacobi wave theory proposed here and the mathematical investigation of some of its physical properties are expected to help shedding further light on the long-standing problem of the canonical formulation of General 
Relativity and its corresponding quantum representation, and might provide also the basis for further future theoretical developments on these issues.

Thus, the manifestly-covariant quantum gravity theory stemming from the classical reduced Hamiltonian theory can only apply to describe the physics associated with massive gravitons, namely massive quanta of the gravitational field, while massless gravitons remain excluded from the spectrum of discrete gravitational solutions that can be predicted by the manifestly-covariant quantum gravity theory.

Author Contributions: Investigation, C.C. and M.T.; Methodology, C.C. and M.T.; Writing—original draft, C.C. and M.T.; Writing - review and editing, C.C. and M.T.

Funding: This research received no external funding.

Conflicts of Interest: The authors declare no conflict of interest.

\section{Appendix A. Extended Canonical Equation in 4-Vector Poisson Brackets Notation}

For completeness, we report the representation of the extended canonical equations given above (see Equations (16) and (17)) in terms of local 4-vector Poisson brackets. To this aim, let us consider first two 4-scalars $A$ and $B$ and then two arbitrary tensor fields $\mathbf{A} \equiv A^{\alpha_{1} . \alpha_{n}}$ and $\mathbf{B} \equiv B_{\beta_{1} . . \beta_{m}}$, generally of different order, so that $n \neq m$, and all to be considered smoothly dependent only on the canonical set $x$. Then, respectively for $(A, B)$ and $(\mathbf{A}, \mathbf{B})$, the canonical Poisson brackets are defined in terms of the 4 -vector operators

$$
\begin{aligned}
& {[A, B]_{x, j} } \equiv \frac{\partial A}{\partial g^{\mu v}} \frac{\partial B}{\partial \Pi_{\mu v}^{j}}-\frac{\partial A}{\partial \Pi_{\mu \nu}^{j}} \frac{\partial B}{\partial g^{\mu v}}, \\
& {[\mathbf{A}, \mathbf{B}]_{x, j} \equiv \frac{\partial \mathbf{A}}{\partial g^{\mu v}} \frac{\partial \mathbf{B}}{\partial \Pi_{\mu \nu}^{j}}-\frac{\partial \mathbf{A}}{\partial \Pi_{\mu \nu}^{j}} \frac{\partial \mathbf{B}}{\partial g^{\mu v}}, }
\end{aligned}
$$

where in the second equation the two indices $\mu$ and $v$ saturate in both terms. On the contrary, the index $j$ does not saturate, a feature which is characteristic of the deDonder-Weyl manifestly-covariant theory, in which coordinates and momenta have different tensorial orders. Hence, in the first case the rhs is a 4 -vector, while in the second one the rhs is a tensor of dimension $n+m+1$. The fundamental Poisson brackets holding for the canonical set are therefore

$$
\begin{aligned}
{\left[g^{\beta \gamma}, \Pi_{\beta \gamma}^{\alpha}\right]_{x, j} } & \equiv \frac{\partial g^{\beta \gamma}}{\partial g^{\mu v}} \frac{\partial \Pi_{\beta \gamma}^{\alpha}}{\partial \Pi_{\mu v}^{j}}-\frac{\partial g^{\beta \gamma}}{\partial \Pi_{\mu \nu}^{j}} \frac{\partial \Pi_{\beta \gamma}^{\alpha}}{\partial g^{\mu v}}=\delta_{\mu}^{\beta} \delta_{v}^{\gamma} \delta_{j}^{\alpha}, \\
{\left[g^{\beta \gamma}, g_{\beta \gamma}\right]_{x, j} } & \equiv \frac{\partial g^{\beta \gamma}}{\partial g^{\mu v}} \frac{\partial g_{\beta \gamma}}{\partial \Pi_{\mu v}^{j}}-\frac{\partial g^{\beta \gamma}}{\partial \Pi_{\mu v}^{j}} \frac{\partial g_{\beta \gamma}}{\partial g^{\mu \nu}}=0, \\
{\left[\Pi_{\alpha}^{\beta \gamma}, \Pi_{\beta \gamma}^{\alpha}\right]_{x, j} } & \equiv \frac{\partial \Pi_{\alpha}^{\beta \gamma}}{\partial g^{\mu v}} \frac{\partial \Pi_{\beta \gamma}^{\alpha}}{\partial \Pi_{\mu \nu}^{j}}-\frac{\partial \Pi_{\alpha}^{\beta \gamma}}{\partial \Pi_{\mu \nu}^{j}} \frac{\partial \Pi_{\beta \gamma}^{\alpha}}{\partial g^{\mu v}}=0 .
\end{aligned}
$$

In terms of the fundamental brackets, the Poisson brackets with the Hamiltonian density $H(x, \widehat{x})$ defined by Equation (11) are

$$
\begin{aligned}
{\left[g^{\beta \gamma}, H\right]_{x, j} } & \equiv \frac{\partial g^{\beta \gamma}}{\partial g^{\mu v}} \frac{\partial H}{\partial \Pi_{\mu v}^{j}}=\frac{\partial H}{\partial \Pi_{\beta \gamma}^{j}}, \\
{\left[\Pi_{\beta \gamma}^{\alpha}, H\right]_{x, j} } & \equiv-\frac{\partial \Pi_{\beta \gamma}^{\alpha}}{\partial \Pi_{\mu \nu}^{j}} \frac{\partial H}{\partial g^{\mu \nu}}=-\delta_{j}^{\alpha} \frac{\partial H}{\partial g^{\beta \gamma}} .
\end{aligned}
$$


As a consequence, the continuum canonical Equations (16) and (17) can be equivalently represented in terms of the 4-vector Poisson brackets as

$$
\begin{aligned}
\hat{\nabla}_{\alpha} \Pi_{\mu v}^{\alpha} & =\left[\Pi_{\mu v}^{\alpha}, H\right]_{x, \alpha}, \\
\hat{\nabla}_{\alpha} g^{\mu v} & =\left[g^{\mu v}, H\right]_{x, \alpha} .
\end{aligned}
$$

The fundamental Poisson brackets Equations (A3)-(A5) together with the continuum Hamilton Equations (A8) and (A9) display the Hamiltonian structure characteristic of the extended Hamiltonian theory obtained in the framework of the manifestly-covariant synchronous variational principle.

\section{Appendix B. Admissible Form of Hamilton Principal Function}

As a complementary development to Section 7, in this Appendix, we investigate whether it is possible to give a proof of the functional dependence that is allowed for the function $S_{0}$, which was taken above to be constant. To this aim, we start from considering again the linear combination (88), in which the two 4-scalar functions $S_{t}$ and $S_{k}$ are independent. In order for this representation to be an acceptable solution, it must hold also in the following two limiting cases:

(1) In the limit in which

$$
\lim _{S_{k} \rightarrow 0} S_{2}^{\alpha}=t^{\alpha} S_{t}
$$

This limit is valid, since the remaining contribution $t^{\alpha} S_{t}$ can be alone a solution, as has been proved above.

(2) In the limit in which simultaneously

$$
\lim _{S_{t} \rightarrow 0} S_{2}^{\alpha} \Rightarrow \lim _{S_{k} \rightarrow 0} S_{2}^{\alpha}=0 .
$$

This means that, in the limit in which $S_{t}$ vanishes, $S_{k}$ must also vanish as well, since $k^{\alpha} S_{k}$ alone cannot be a solution, as discussed in Section 6 .

Let us turn our attention again to the functional form of the Hamilton principal functions. We must admit $S_{t}$ to be a generic function of the type $S_{t}=S_{t}(g, \widehat{g}, r, s)$ to be solved by the Hamilton-Jacobi equation. Instead, for $S_{k}$, we have imposed that the variable separation (92) applies, namely that it is possible to write in general

$$
S_{k}=S_{o}(g, \widehat{g}, s) f\left(k_{\mu} r^{\mu}, \widehat{g}\right) \text {. }
$$

This choice allows one to represent the wave dependence associated with $S_{k}$. If we calculate back the conjugate momentum $\Pi_{\alpha}^{\mu v}=\frac{\partial S_{2 \alpha}}{\partial g_{\mu \nu}}$ using this representation, we get

$$
\Pi_{\alpha}^{\mu v}=t_{\alpha} \frac{\partial S_{t}}{\partial g_{\mu v}}+k_{\alpha} f\left(k_{\mu} r^{\mu}, \widehat{g}\right) \frac{\partial S_{o}(g, \widehat{g}, s)}{\partial g_{\mu v}} .
$$

Then, the extended Hamilton Equations (16) and (17) yield

$$
\begin{aligned}
\hat{\nabla}_{\alpha} \Pi_{\mu \nu}^{\alpha} & =t^{\alpha} \widehat{\nabla}_{\alpha} \frac{\partial S_{t}}{\partial g^{\mu \nu}}+k^{\alpha} \frac{\partial S_{o}(g, \widehat{g}, s)}{\partial g^{\mu \nu}} \widehat{\nabla}_{\alpha} f\left(k_{\mu} r^{\mu}, \widehat{g}\right)=-\frac{\partial H(x, \widehat{x})}{\partial g^{\mu \nu}}, \\
\hat{\nabla}_{\alpha} g^{\mu \nu} & =\frac{1}{\kappa}\left(t_{\alpha} \frac{\partial S_{t}}{\partial g_{\mu v}}+k_{\alpha} f\left(k_{\mu} r^{\mu}, \widehat{g}\right) \frac{\partial S_{o}(g, \widehat{g}, s)}{\partial g_{\mu v}}\right) .
\end{aligned}
$$


In the first equation, we use again the wave-like dependence carried by $f\left(k_{\mu} r^{\mu}, \widehat{g}\right)$ and the fact that $k^{\alpha} k_{\alpha}=0$, so that we can reduce the previous equations to the simplified form

$$
\begin{aligned}
t^{\alpha} \hat{\nabla}_{\alpha} \frac{\partial S_{t}}{\partial g^{\mu v}} & =-\frac{\partial H(x, \widehat{x})}{\partial g^{\mu v}} \\
\hat{\nabla}_{\alpha} g^{\mu v} & =\frac{1}{\mathcal{K}} t_{\alpha} \frac{\partial S_{t}}{\partial g_{\mu v}}+\frac{1}{\mathcal{K}} k_{\alpha} f\left(k_{\mu} r^{\mu}, \widehat{g}\right) \frac{\partial S_{o}(g, \widehat{g}, s)}{\partial g_{\mu v}} .
\end{aligned}
$$

From Equation (A17), we notice in particular that the differential contribution due to $S_{t}$ is analogous to that due to $S_{0}$. Therefore, from the information derived from the Hamilton equations, we can say that the limiting condition (A11) required for the validity of the solution (88) becomes now

$$
\lim _{S_{t} \rightarrow 0} \frac{\partial S_{t}}{\partial g_{\mu \nu}}=\lim _{S_{o} \rightarrow 0} \frac{\partial S_{o}}{\partial g_{\mu v}}=0 .
$$

Since $S_{o}$ is undetermined, the previous condition requires that it is either

$$
S_{o}=S_{o}\left(S_{t}\right)
$$

namely $S_{o}$ depends functionally on $S_{t}$ and they vanish jointly, or that it is separately

$$
S_{o}=\text { const }
$$

However, the formal solution (A19) must be excluded ad absurdum, since it contradicts the initial hypothesis (A12) on the functional dependence of the scalar function and the variable separation, as generally one has that $S_{t}=S_{t}(g, \widehat{g}, r, s)$ and for $S_{t}$ no analogous variable separation exists. In addition, it contradicts also the initial hypothesis introduced in Equation (88), when in the linear combination the two functions $S_{t}$ and $S_{k}$ are taken to be independent from each other.

In conclusion, the only choice that warrants validity of the representation (A11) is by selecting the 4-scalar function $S_{k}$ as

$$
S_{k}=S_{o} f\left(k_{\mu} r^{\mu}, \widehat{g}\right),
$$

with $S_{o}=$ const. necessarily. In this context, the function $S_{k}$ represents a sort of gauge function, which however remain always in the classical domain and does not contribute to the corresponding quantum solution. The simplest type of representation of the function $f\left(k_{\mu} r^{\mu}, \widehat{g}\right)$ is in terms of a plane wave, although more general expressions that differ from the planar wave case can be in principle accounted for through suitably setting the dependence in terms of $k_{\mu} r^{\mu}$.

\section{References}

1. De Donder, T. Théorie Invariantive Du Calcul des Variations; Gaultier-Villars \& Cia.: Paris, France, 1930.

2. Weyl, H. Geodesic Fields in the Calculus of Variation for Multiple Integrals. Ann. Math. 1935, 36, 607. [CrossRef]

3. Saunders, D.J. The Geometry of Jet Bundles; Cambridge University Press: Cambridge, UK, 1989.

4. Sardanashvily, G. Generalized Hamiltonian Formalism for Field Theory; World Scientific Publishing: Singapore, 1995.

5. Echeverría-Enríquez, A.; Muñoz-Lecanda, M.C.; Román-Roy, N. Geometry of Lagrangian first-order classical field theories. Fortschr. Phys. 1996, 44, 235. [CrossRef]

6. Kanatchikov, I.V. Canonical structure of classical field theory in the polymomentum phase space. Rep. Math. Phys. 1998, 41, 49. [CrossRef]

7. Forger, M.; Paufler, C.; Romer, H. The Poisson bracket for Poisson forms in multisymplectic field theory. Rev. Math. Phys. 2003, 15, 705. [CrossRef]

8. Kisil, V.V. p-Mechanics as a physical theory: An introduction. J. Phys. A Math. Gen. 2004, 37, 183. [CrossRef] 
9. Struckmeier, J.; Redelbach, A. Covariant Hamiltonian field theory. Int. J. Mod. Phys. E 2008, $17,435$. [CrossRef]

10. Cremaschini, C.; Tessarotto, M. Synchronous Lagrangian variational principles in General Relativity. Eur. Phys. J. Plus 2015, 130, 123. [CrossRef]

11. Dirac, P.A.M. Generalized Hamiltonian Dynamics. Can. J. Math. 1950, 2, 129. [CrossRef]

12. Sundermeyer, K. Constrained Dynamics; Lecture Notes in Physics; Springer: Berlin, Germany, 1982.

13. Einstein, A. The Meaning of Relativity; Princeton University Press: Princeton, NJ, USA, 2004.

14. Landau, L.D.; Lifschitz, E.M. Field Theory, Theoretical Physics; Addison-Wesley: New York, NY, USA, 1957; Volume 2.

15. Misner, C.W.; Thorne, K.S.; Wheeler, J.A. Gravitation, 1st ed.; W.H. Freeman: Princeton, NJ, USA, 1973.

16. Tessarotto, M.; Cremaschini, C. Theory of Nonlocal Point Transformations in General Relativity. Adv. Math. Phys. 2016, 2016, 9619326. [CrossRef]

17. Jordi, G.; Narciso, R.R. Multisymplectic unified formalism for Einstein-Hilbert gravity. J. Math. Phys. 2018, 59, 032502.

18. Cremaschini, C.; Tessarotto, M. Manifest covariant Hamiltonian theory of General Relativity. Appl. Phys. Res. 2016, 8, 2. [CrossRef]

19. Cremaschini, C.; Tessarotto, M. Hamiltonian approach to GR-Part 1: Covariant theory of classical gravity. Eur. Phys. J. C 2017, 77, 329. [CrossRef]

20. Cremaschini, C.; Tessarotto, M. Hamiltonian approach to GR-Part 2: Covariant theory of quantum gravity. Eur. Phys. J. C 2017, 77, 330. [CrossRef]

21. Cremaschini, C.; Tessarotto, M. Quantum-wave equation and Heisenberg inequalities of covariant quantum gravity. Entropy 2017, 19, 339. [CrossRef]

22. Tessarotto, M.; Cremaschini, C. Generalized Lagrangian path approach to manifestly-covariant quantum gravity theory. Entropy 2018, 20, 205. [CrossRef]

23. Tessarotto, M.; Cremaschini, C. Role of quantum entropy and establishment of H-theorems in the presence of graviton sinks for manifestly-covariant quantum gravity. Entropy 2019, 21, 418. [CrossRef]

24. Cremaschini, C.; Tessarotto, M. Space-time second-quantization effects and the quantum origin of cosmological constant in covariant quantum gravity. Symmetry 2018, 10, 287. [CrossRef]

25. Arnowitt, R.; Deser, S.; Misner, C.W. Gravitation: An Introduction to Current Research; Witten, L., Ed.; Wiley: New York, NY, USA, 1962.

26. Etienne, Z.B.; Liu, Y.T.; Shapiro, S.L. Relativistic magnetohydrodynamics in dynamical spacetimes: A new AMR implementation. Phys. Rev. D 2010, 82, 084031. [CrossRef]

27. Alcubierre, M. Introduction to 3+1 Numerical Relativity; Oxford University Press: Oxford, UK, 2008.

28. Gheorghiu, T.; Vacaru, O.; Vacaru, S. Off-diagonal deformations of kerr black holes in Einstein and modified massive gravity and higher dimensions. Eur. Phys. J. C 2014, 74, 3152. [CrossRef]

29. Messiah, A. Quantum Mechanics; Dover Pubs: New York, NY, USA, 1999.

30. Cremaschini, C.; Tessarotto, M. Quantum theory of extended particle dynamics in the presence of EM radiation-reaction. Eur. Phys. J. Plus 2015, 130, 166. [CrossRef]

31. Tessarotto, M.; Cremaschini, C. Generalized Lagrangian-path representation of non-relativistic quantum mechanics. Found. Phys. 2016, 46, 1022-1061. [CrossRef]

32. Goldstein, H. Classical Mechanics, 2nd ed.; Addison-Wesley: Reading, MA, USA, 1980.

(C) 2019 by the authors. Licensee MDPI, Basel, Switzerland. This article is an open access article distributed under the terms and conditions of the Creative Commons Attribution (CC BY) license (http://creativecommons.org/licenses/by/4.0/). 